\title{
The Star Formation History of Galaxies Measured from Individual Pixels. I. The Hubble Deep Field North
}

\author{
Alberto Conti ${ }^{1}$, Andrew J. Connolly, Andrew M. Hopkins ${ }^{2}$ \\ Department of Physics and Astronomy, University of Pittsburgh, \\ 3941 O’Hara Street, Pittsburgh, PA 15260 \\ Tamas Budavári, Alex S. Szalay, Istvàn Csabai \\ Department of Physics and Astronomy, John Hopkins University, \\ 3400 North Charles Street, Baltimore, MD, 21218 \\ Samuel J. Schmidt, Carla Adams ${ }^{3}$, Nada Petrovic ${ }^{4}$ \\ Department of Physics and Astronomy, University of Pittsburgh, \\ 3941 O’Hara Street, Pittsburgh, PA 15260
}

Received __; accepted _ _

\footnotetext{
${ }^{1}$ Currently at Computer Sciences Corporation, Space Telescope Science Institute, 3700 San Martin Drive, Baltimore, MD 21218

${ }^{2}$ Hubble Fellow

${ }^{3}$ Currently at the New Mexico State University, MSC 4500 Astronomy P.O. Box 30001, Las Cruces, NM 88003

${ }^{4}$ Currently at the Department of Astronomy and Astrophysics, 5640 S. Ellis Ave, Chicago, IL 60637
} 


\begin{abstract}
We analyze the photometric information contained in individual pixels of galaxies in the Hubble Deep Field North (HDFN) using a new technique, pixel$z$, that combines predictions of evolutionary synthesis models with photometric redshift template fitting. Each spectral energy distribution template is a result of modeling of the detailed physical processes affecting gas properties and star formation efficiency. The criteria chosen to generate the SED templates is that of sampling a wide range of physical characteristics such as age, star formation rate, obscuration and metallicity. A key feature of our method is the sophisticated use of error analysis to generate error maps that define the reliability of the template fitting on pixel scales and allow for the separation of the interplay among dust, metallicity and star formation histories. This technique offers a number of advantages over traditional integrated color studies. As a first application, we derive the star formation and metallicity histories of galaxies in the HDFN. Our results show that the comoving density of star formation rate, determined from the UV luminosity density of sources in the HDFN, increases monotonically with redshift out to at least redshift of 5 . This behavior can plausibly be explained by a smooth increase of the UV luminosity density with redshift coupled with an increase in the number of star forming regions as a function of redshift. We also find that the information contained in individual pixels in a galaxy can be linked to its morphological history. Finally, we derive the metal enrichment rate history of the universe and find it in good agreement with predictions based on the evolving HI content of Lyman- $\alpha$ QSO absorption line systems.
\end{abstract}

Subject headings: cosmology: observations - galaxies: distances and redshifts - galaxies: evolution — galaxies: formation — galaxies: luminosity function 


\section{Introduction}

In recent years the photometric redshift technique has become a viable and cheap alternative to spectroscopic redshifts (Loh \& Spillar 1986; Connolly et al. 1995; Lanzetta, Yahil, \& Fernández-Soto 1996; Gwyn \& Hartwick 1996; Mobasher et al. 1996; Sawicki et al. 1996; Balzonella et al. 2000; Furusawa et al. 2000; Budavári, et al. 2001; Fernández-Soto et al. 2001; Richards et al. 2001; Le Borgne \& Rocca-Volmerange 2002). In all those cases where galaxies are too faint for spectroscopic studies, the photometric redshift technique remains the only practical way of estimating galaxy redshifts. With ongoing large multicolor observational programs such as the Sloan Digital Sky Survey and 2MASS, the photometric redshift technique will prove to be an even more powerful tool in interpreting these increasingly large and detailed data sets. The use of photometric redshifts will open the door to the study of multivariate distributions (such as the galaxy luminosity function) and may in fact provide statistical redshift distributions for any population of galaxies in any environment (Schmidt 2003, in preparation).

Here we focus on what is perceived by many as a byproduct of the photometric redshift technique, namely the best fitting spectral energy distribution (SED) template. This work takes as its starting point a new approach in the use of the photometric redshift technique that extends and expands its original purpose. Our motivation resides in recent technical and conceptual developments that make this new step possible. On one hand, the use of sophisticated training algorithms (Budavári, et al. 2000) allows for a better interpretation of the intrinsic photometric properties of a sample. This step shifts the focus from the photometric redshift technique in itself to the actual properties of the sample. Secondly, deep surveys such as the Hubble Deep Fields ${ }^{5}$ (Williams at al. 1996, 2000) have stimulated

\footnotetext{
${ }^{5}$ Based on observations with the NASA/ESA Hubble Space Telescope obtained at the Space Telescope Science Institute, which is operated by the Association of Universities for
} 
the growth of ideas, new techniques and "proof of concept" papers. One of these papers (Abraham et al. 1999, hereinafter ABR99), focused on "exploring the resolved multicolor data for galaxies of known redshift using spectral-synthesis models" thereby dramatically shifting the focus of the photometric redshift technique to the reliability of the template spectra used in the fitting.

Our work takes ABR99 as a starting point. However, instead of focusing as ABR99 did on the understanding of the relative ages of bulges and disks, and the formation history of elliptical galaxies via spatially resolved colors, we focus onto two direct enhancements of the ABR99 technique and two distinct applications:

$\triangleright$ Use of all available redshifts: ABR99's sample was, at the time, the faintest statistically-complete spectroscopic redshift sample currently available in the HDFN. We extend his sample by including not only the $\sim 190$ object in the HDFN with measured spectroscopic redshifts, but all of the $\sim 1500$ galaxies detected in the HDFN for which we will measure photometric redshifts. While this approach propagates the larger redshift uncertainties onto the measured properties of galaxies, the use of the original HDFN passbands extended by the NICMOS data reduces the redshift uncertainty to more than acceptable levels $\sigma_{z} \sim 0.05$ (Connolly et al. 1997). Furthermore, as we will show in Section 4, Monte Carlo realizations of the photometric redshift uncertainty on all galaxies in the HDFN with measured spectroscopic redshift, show that the derived properties of galaxies are not adversely affected by uncertainties in redshift as large as $\sigma_{z}=0.2$.

$\triangleright$ Wide template range: By extending the 4-filter color approach of ABR99 to a 6-filter 2160 template approach, we allow for a more detailed description of the individual 
characteristics of the object being compared to the set of templates. In particular our description will allow for variations in age, dust content, metallicity and star formation rate on a wide range of scales. As a consequence of this "redundant" approach, we will be able to resolve subtle differences within an individual galaxy's stellar populations. This is one of the goals of our approach. In addition, the large number of templates used will allow for a multivariate error analysis on a pixel-by-pixel level. This in turn will produce detailed error maps for each HDFN galaxy. We will make use of such maps to assess the accuracy and the significance of the physical characteristics derived from each pixel's SED.

- Science from individual pixels: Each pixel can be regarded as the smallest "multicolor unit" available in the HDFN. We will make use of the multicolor information for each of the pixels in a galaxy to constrain the relative ages for physically distinct sub-components of the galaxy and determine their dust and metallicity content. As a byproduct, in Section 2 we will also be able to determine which pixel, and hence which parts of a galaxy, are contributing to the estimate of the photometric redshift. This feature has the potential of being able to separate projected objects that are close only in angular distance.

- Comoving density of star formation and metals: The comoving density of star formation and metallicity enrichment are calculated as a function of redshift using individual pixels in the HDFN. We will compare the results obtained by other groups using standard techniques with our own approach. In addition, we will compute the luminosity function of galaxies in redshift intervals, integrate it out to faint magnitudes and recover the luminosity function correction to be applied to our derived star formation rates (SFR) to compensate for the way different magnitude ranges are sampled at different redshifts. 
The outline of the paper is as follows. In Section 2 we will illustrate the pixel-z method and why we have decided for its first application on the Hubble Deep Field North. In Section 3 we describe the strategy and trade offs necessary to create a set of template spectral energy distributions that cover a wide range of physical characteristics. Section 4 presents the results of the pixel-z method including the maximum likelihood error analysis we used to disentangle uncertainties and degeneracies introduced by the use of a large set of templates. In this section we will also compute, based on our findings, the star formation rate of all pixels in the HDFN. In Section 5 we calculate the star formation history of all pixels in the HDFN. Furthermore, we derive the luminosity function in redshift bins to account for the large fraction of missing luminosity at high redshift. Finally, we calculate the comoving metal enrichment rate for all pixels in the HDFN. Section 6 presents the conclusions.

Throughout the manuscript, we assume a matter dominated universe with $\Omega_{m}=1$ and $H_{0}=75 h \mathrm{~km} \mathrm{~s}^{-1} \mathrm{Mpc}^{-3}$.

\section{The Pixel-z Method}

By making use of strong spectral features such as the $4000 \AA$ break, the Balmer break and the Lyman decrement, the standard template-fitting photometric redshift techniques is able to return a fast and accurate estimate of a galaxy's redshift. Generally, the fitting function takes the following form:

$$
\chi^{2}(z, T)=\sum_{i=1}^{N_{\mathrm{f}}} \frac{\left[F_{\mathrm{obs}, i}-b_{j} \times F_{i, j}(z, T)\right]^{2}}{\sigma_{i}^{2}}
$$

where $F_{\text {obs }, i}$ is the flux through the ith filter, $b_{j}$ is a scaling factor, $F_{i, j}$ is the flux through the ith filter of the jth spectral energy distribution template (calculated at a redshift $z$ ) and $\sigma_{i}$ is the uncertainty in the observed flux. The sum is carried out over all available filters 
$N_{\mathrm{f}}$. The resulting $\chi^{2}$ is then minimized as a function of template $T$ and redshift $z$ providing an estimate of the redshift of the galaxy and its spectral type (together with the variance on these measures).

The accuracy of the photometric redshift estimate obtained by applying equation (1) to galaxy data, critically depends on the number and wavelength coverage of the passband filters used, and on the signal-to-noise characteristics of the source. At low redshifts $(z \lesssim 0.7)$ it is important to cover the ultra-violet and optical region of the spectrum in order to be able to "bracket" the $4000 \AA$ break with the U, B and V passbands. At very high redshifts $(2.5<z<5.0)$ the ultra-violet region becomes very important as the $912 \AA$ break is shifted into the $U_{300}$ passband filter (Madau et al. 1996; Steidel et al. 1996). At intermediate redshifts adequate coverage of the spectral features is guaranteed by infrared filters: the $\mathrm{J}$ filter being instrumental for galaxies at $z \lesssim 1.5$, while the $\mathrm{H}$ and $\mathrm{K}$ filters extend the redshift coverage past $z \sim 2.5$. Provided UV and IR filters are available, at least four filters are needed to identify the strong spectral features required to obtain a statistically significant estimate of the redshift. However, modern deep surveys can boost this number to as many as six, thereby significantly improving the reliability of the redshift estimates at any redshift (Hogg et al. 1998).

Generally, the observed flux $F_{\text {obs }, i}$ is taken to be that of the entire galaxy in each bandpass obtained by adding the contribution of all pixels (identified as being part of the galaxy) above some chosen threshold ${ }^{6}$. Commonly used object detection software packages, in fact, not only identify and catalog objects, but also produce a list of pixels belonging to each of the identified objects. This catalog of pixels for each galaxy will be the starting point of our analysis.

\footnotetext{
${ }^{6}$ Typically an arbitrary $3 \sigma$ cut above sky level is chosen, but see also Hopkins et al. (2001) for a more rigorous definition of detection threshold.
} 
The information required to estimate the photometric redshift of an object does not make direct use of the pixel information and is limited to the objects' observed magnitudes and relative measurement errors. However, the flux of individual pixels could, in principle, be treated as a separate "source" within the data and, thanks to the quite general form of the fitting function given by equation (1), a photometric redshift for individual pixels can be computed. ${ }^{7}$

While the extension of the photometric redshift technique to individual pixels is straightforward (modulo the photometric error propagation), the real issue resides in the interpretation of the results. Can the use of photometric redshifts computed on individual pixels provide new insights into the intrinsic properties of galaxies? Moreover, once a photometric redshift for a pixel inside a galaxy has been determined, what is its significance in terms of the global photometric or spectroscopic redshift of the entire galaxy? Finally, how does the photometric redshift error propagate on a pixel scale?

This work addresses all the aforementioned questions. In particular, we have developed a new technique called pixel-z to take advantage of the information contained in individual pixels that

1. enables the deblending of systems associated by angular superposition alone;

\footnotetext{
${ }^{7}$ We should mention that the analysis of galaxy images pixel by pixel has the potential disadvantage of introducing larger photometric uncertainties than total fluxes measured in apertures. Ideally one would like to keep the spatial information present in the image along with a better signal-to-noise ratio. A possible solution is described in Budavári, et al. (2003), where spatially connected pixels of similar colors are joined into superpixels in order to improve on the statistical errors without mixing the different galaxy components, e.g. the red bulge or the bluer star-forming regions in spiral arms.
} 
2. enables one to decompose the internal photometric structure of observed galaxies into basic constituents such as the age of the stellar population, their metallicities and their dust content;

3. enables, under simplifying assumptions, the determination of the star formation rate for individual pixels inside a galaxy;

4. returns the contribution of each pixel to the star formation history of a galaxy thereby allowing, for a sufficiently large sample of galaxies, a direct assessment of the drivers behind the current understanding of the global star formation history plot.

For the first application of this technique, we choose to use the Hubble Deep Field North (HDFN). This rich dataset has many of the characteristics that make it an ideal testbed for our purposes. First and foremost, it is among the best studied areas of the sky and, as such, can be regarded an ideal benchmark for our technique. Moreover, the HDFN provides not only one of the deepest images for multi-color selection of "Lyman break" galaxies but also morphological and size information for high redshift galaxies. For our analysis we will make use of the $2500 \times 2500$ pixel versions of the HDFN and NICMOS data together with the relative weight maps. The latter are needed to correctly estimate the photometric errors on individual pixels. We use version 1.04 of the renormalized rms maps. These maps give the "true" noise level in the data, corrected for inter-pixel correlations. These correlations, induced both by drizzling and by the use of convolution kernels during the reduction process, reduce the apparent pixel-to-pixel noise level in the images, making it difficult to assess flux uncertainties without knowing the correction factors. With the rms images, one can sum (in quadrature) the noise values for pixels over any aperture and determine the effective flux uncertainty within that aperture or use the measure on a pixel-by-pixel basis. The rms maps quantify the uncertainty due to noise in the background. These maps are used to determine the detection significance of an object or a pixel. We 
shall return to this point later on when we will show how the error in the pixels of an object will allow for the construction of an object's error map used to assess the significance of our photometric decomposition.

The results of the application of the photometric redshift technique to individual pixels in galaxies in the HDFN is shown in Figure 1 and Figure 2. For each pixel a photometric redshift is computed using the template fitting technique. The number of templates used in this particular case is rather small, being the four empirical SEDs compiled by Coleman et al. (1980) (hereafter CWW). However, as we shall see later on, this does not change the reliability of this particular part of our analysis. For each of the pixels, the resulting photometric redshift estimate is simply a function of the flux in each of the available band-passes. When applied to the entire galaxy (i.e. the sum of the contributions from individual pixels), the photometric redshift returned should correlate with the number of pixels that have photometric redshifts near the galaxy overall redshift. For the galaxy in Figure 1, the measured spectroscopic redshift is $z=1.013$, while the measured photometric redshift is $z=1.061 \pm 0.05$. The galaxy shown is rather bright $(F 160 W \sim 19.88)$, and most of the pixels in the galaxy appear tightly distributed around $z \sim 1$, peaking at a redshift just greater than one with a rather tight $F W H M=0.11$. This value of the FWHM is typical of galaxies in the HDFN. In fact, almost all galaxies in the HDFN display this tight unimodal distribution. The entire sample is characterize by a typical $F W H M=0.16$.

While this behavior was somehow expected by "construction", perhaps the more interesting features reside in the two other peaks at higher redshift and the information they might contain. One can envision a scenario in which two galaxies at different redshifts, but close in angular distance, might be separated by using this method. We will address this issue in a forthcoming paper. In this particular case, the two "extra" peaks do not belong to separate objects but are a direct result of the lower signal-to-noise ratio of the 
outskirts of the galaxy, which in turn are best fitted by SEDs at much higher redshift.

Figure 2 on the other hand, displays a different behavior. This galaxy appears in the HDFN images as either two or possibly three separate objects. The distribution of pixels reflects this multiple object hypothesis. Three peaks of comparable height are clearly recognizable at redshift $z \sim 0, z \sim 0.85, z \sim 3$. The template distribution shows that either we are in the presence of a single object at spectroscopic redshift $z=0.130$ (Dickinson et al. 2003) with distinct and spatially separated stellar populations, or we are in the presence of separate objects that have been erroneously identified as a single galaxy. The latter hypothesis is strongly supported by direct comparison with the work of Conti et al. (1999). In fact, the component at $z \sim 0$ has been identified by Conti et al. (1999) as the most likely low redshift quasar candidate in the HDFN (ID0094). This suggests that we are in the presence of at least 2 objects, one of which has been correctly identified via template fitting as "stellar-like".

These simple examples portray the extended reach of this technique. Our present work, however, takes Figures 1 and 2 as our starting point and focuses on the relative properties of each galaxy by fixing the redshift of all pixels to be that of the whole galaxy, determined either photometrically or spectroscopically. This approach is equivalent to removing the redshift dependence in equation (1) and solving for the best fitting template type. Removing the redshift dependence, however, does not eliminate the existing covariance between redshift and SED template.

Generally, the resulting best fitting template is treated as a byproduct of the photometric redshift estimate, the redshift being the focus of the fitting. This is in part due to the prevalent small number of templates used (typically local galaxy data and hence not entirely representative of the large variety of SEDs observed at different epochs), and in part to the use of evolutionary synthesis models which remain untested outside the 
standard Hubble types.

The current implementation of the pixel-z technique, shifts the attention to the SED templates. In fact, the SED of a galaxy should reflect the distribution of stellar masses, ages and metallicities and hence provide clues to the past history of star formation. Building on this fact, we will show in the next Sections that, by fitting SEDs to individual pixels in a galaxy, we can recover the morphological characteristics of the galaxy and, perhaps more importantly, separate the individual contributions of age, metallicity, dust and star formation history.

\section{Template Construction}

We use the most recent Bruzual \& Charlot (2000) models to generate a large number of SEDs that will be used for template fitting for each of the pixels of all the HDFN galaxies. Each SED is a result of modeling of the detailed physical processes affecting gas properties and star formation efficiency. The modeling is generally based on assumptions about the stellar birthrate. The adjustable parameters in the models are typically the initial stellar mass function (IMF), the star formation rate and chemical enrichment.

The criteria we have chosen to generate the SED templates is that of maximizing our ability to solve for the aforementioned quantities. Specifically, we generate SEDs with the following characteristics:

1. we allow the underlying stellar population within each pixel to vary over a wide age range. Typically from extremely young (0.001,0.01,0.1,0.5 Gyrs), to middle age (1,3,5 Gyrs), to old and very old $(9,12,15$ Gyrs), for a total of ten ages;

2. we assume that the fluxes of individual pixels can be modeled using an exponential star-formation rate with an e-folding timescale $\tau$, i.e. $\Psi(t)=\Psi_{0} e^{(-t / \tau)}$. This 
parametrization turns out to be quite convenient for its simplicity in describing the star formation rate of an instantaneous burst when $\tau \rightarrow 0$ and a constant star formation when $\tau \rightarrow \infty$. The e-folding times used for $\tau$ are 0.1 Gyrs for an extremely short bursts, 1,3,5,9 and 12 Gyrs for subsequently longer bursts. Is is worth noting at this point that an exponential star formation rate for individual pixels does not inevitably lead to an exponentially decaying star formation rate for the galaxy as a whole other than in the special case in which every pixel in the galaxy is coeval and all have the same star-formation rate;

3. since pixels with any star formation history can be expanded in series of instantaneous bursts, each having fixed metallicity, the spectral evolution of individual pixels (or whole galaxies) can be investigated without prior knowledge of chemical evolution. We assume the SEDs to be characterized by six possible metallicities, ranging from $\frac{1}{50}$ to 2.5 times that of the sun;

4. the general spectral characteristics of the SEDs of galaxies will be modified by the presence of dust. We parametrize dust obscuration in terms of the relative optical extinction in the rest frame $E_{B-V}$ using the reddening curve $k(\lambda)=A(\lambda) / E_{B-V}$ for star-forming systems formulated by Calzetti et al. (2000). For each of the SEDs we allow for six independent values of extinction ranging from no extinction to 0.9 magnitudes of extinction.

In all our templates, we assumed a Salpeter IMF with low and high mass cut-offs of $0.1 M_{\odot}$ and $125 M_{\odot}$ respectively. 


\section{Analysis}

The majority of the galaxies in the HDFN do not have measured spectroscopic redshifts. These, in fact, amount only to $\sim 190$, while the total number of galaxies cataloged is $\sim 1500$. Therefore, we make use of equation $(1)$ together with the CWW templates to determine the redshift of all galaxies in the HDFN. We then generate SEDs that span 10 ages, 6 star formation e-folding times, 6 metallicities and 6 color extinctions for a total of 2160 templates. We fit each of the 2160 SED templates to all the pixels in each of the HDFN galaxies maintaining the redshift of all pixels fixed to that of their host galaxy during the entire procedure. This latter step effectively removes one of the degrees of freedom in

our fit and returns the properties of each of the pixels in terms of their best fitting template.

The best fitting template is calculated using a least squares maximum likelihood estimator similar in spirit to the one of equation (1), but with a much larger number of SED templates, specifically:

$$
\mathcal{L}(\mathcal{T})=\prod_{n=1}^{N_{\mathrm{f}}} \frac{1}{\sqrt{2 \pi} \sigma_{i}} \exp \left\{-\frac{\left[F_{\text {obs }, i}-b_{j} \times F_{i, j}\left(z_{\text {gal }}, \mathcal{T}\right)\right]^{2}}{2 \sigma_{i}{ }^{2}}\right\}
$$

where the SED templates $\mathcal{T}=\mathcal{T}\left\{t, \tau, E_{B-V},[F e / H]\right\}$ now explicitly accounts for the dependence on age, e-folding time, extinctions and metallicity. The redshift of all pixels $z_{\text {gal }}$ is held fixed. Before we can interpret the results of this fit, however, we need to state clearly the assumptions and simplifications we make while computing the fit.

Firstly, we make the simplifying assumption that the all pixels in the galaxy are coeval, that is, we explicitly assume that the galaxy as a whole, and hence each pixel within it, has a common age. This assumption is not required by our analysis and is not a limitation of our approach. Instead it serves as a simplifying starting point for the first application of the pixel-z technique. The actual "ages" being fit to in each pixel in a galaxy are a result of the luminosity-weighted ages of the stellar populations probed within the pixel, that produce 
the observed pixel colors. The "age" referred to by the synthesis model used, though, is simply the time since the onset of initial star formation in the model. The relationship for a passively-evolving system is straightforward, but for a galaxy where knots of recent star-formation are visible, or which has experienced a complex star formation history, the relationship is much less clear. The assumption of a common age for all pixels in a whole galaxy, then, can be seen to be somewhat over-simplistic, but it serves as an important first step in evaluating the effectiveness of the pixel-z technique, and greatly simplifies the interpretation of the results of the remaining fitted parameters on a galaxy-by-galaxy basis. Future applications of this technique will not be limited by this assumption.

Each of the SEDs we use is simply a realization of the 4 dimensional space of age, e-folding time, extinction and metallicity. To determine the "best fitting age" of all the pixels in the galaxy, we use equation (2) to marginalize our likelihood estimator over the age of the stellar population in each pixel. By multiplying the likelihoods of all pixels for each of the 10 modeled ages, we determined a unique value for the likelihood of the entire galaxy at any given age. This likelihood is then maximized and the best fitting age is determined. This assumption, while not strictly consistent with galaxy formation scenarios, has the advantage of providing greater ease of interpretation of the remaining parameters in the context of the whole galaxy, in particular the star formation history of the system. We stress here that we could have chosen not to make this assumption, but our main concern at this stage of development of the pixel-z technique is to understand what the best fitting template returned for each pixel is able to tell us about the underlying physical conditions of the galaxy. We will relax this assumption in a subsequent investigation.

Secondly, while simple to parametrize in the SEDs, the e-folding time of an exponentially decaying star formation is not directly reconcilable with the star formation rate per pixel, which would be a much more desirable quantity to extract from the SEDs. 
To determine the star formation rate per pixel in physical units, we use the empirical formula of Kennicutt (1998) to link the UV luminosity of each pixel to its intrinsic star formation rate:

$$
\operatorname{SFR}\left(M_{\odot} y r^{-1}\right)=1.4 \times 10^{-28} L_{\nu}\left(\operatorname{ergs~s}^{-1} \mathrm{~Hz}^{-1}\right)
$$

We implicitly assume that this calibration holds at all redshifts. Given the fact that the redshift of each pixel has been fixed to that of its host galaxy, a pixel's absolute UV luminosity $L_{\nu}$ can be easily computed for any cosmological model, after incorporating the necessary K-correction. However, for our estimate to be reliable, we need to assess how $L_{\nu}$ determined from aperture photometry for the entire galaxy compares to that of all pixels added together. The difference could be particularly noticeable for galaxies in which a fixed aperture measures only their inner flux. This comparison has the dual purpose of calculating the correction factor to be applied to each pixel to reconcile the measurement of the UV luminosities, and also to provide a scaling factor to be applied to all pixels to convert the star formation rate returned by Bruzual \& Charlot (2000) into physical units. The spectrophotometric synthesis models of Bruzual \& Charlot (2000), in fact, return a star formation rate normalized to a $1 M_{\odot}$ galaxy. The results of this comparison are discussed below.

\subsection{Global vs Local Star Formation Rate}

We use the publicly available HDFN photometric catalog (Williams at al. 1996) together with either spectroscopic or photometric redshift estimates, to determine each galaxy's absolute UV luminosity. We then make use of equation (3) to determine a galaxy's star formation rate. Comparison with the star formation rate determined in a similar fashion for all the pixels in a galaxy yields a normalization constant, which in turn is

used to calculate the star formation rate in $M_{\odot} y r^{-1}$ for individual pixels. As pointed out 
by our referee, we could have avoided the computational steps required to calculate this normalization constant by making direct use of the output of the Bruzual and Charlot models. We updated our pipeline to make use of this direct approach. To ensure consistency, we also ran a series of tests to determine to what extent our global normalization would differ from that obtained directly by the Bruzual and Charlot's models. Our tests revealed this discrepancy to be of the order of a few percent and suggest that for all practical purposes the two methods indeed are equivalent.

In Figure 3 the top panel shows on the abscissa the star formation rate computed from aperture photometry for all galaxies in the HDFN versus the star formation rate derived from the sum of all pixels in the same galaxies. The line represents the one-to-one relation and is not a fit to the data. The rms is 0.86 . The agreement is quite good, even though the pixel-z result seems to slightly overestimate the overall SFR. This behavior can be understood, as we mentioned earlier, by noticing that for large galaxies a fixed aperture might not include all pixels.

The bottom panel shows the residual uncertainties in the measurement of the star formation rate. Once again, aside from a few outliers, the scatter is sufficiently small that we are confident our estimate of the galaxy's UV luminosity obtained by adding the contribution of individual pixels is indeed consistent with aperture photometry measurements. For those galaxies with no UV detection, we have assumed an upper limit for their fluxes as determined from the $U_{300}$ rms maps. These objects are generally small and, when using aperture photometry, the aperture is likely to include many background pixels, which in turn give the appearance of larger star formation rates. These outliers are clearly visible in the residual plot.

The validity of the agreement displayed in Figure 3 will be tested further when we calculate the star formation history of all the galaxies in the HDFN and compare it to 
known results from the literature.

\subsection{Galaxy Maps}

The result of the fit of the 2160 SED templates to a large spiral in the HDFN is shown in Figure 4. The top left map shows the galaxy in the F606W WFPC2 filter. The other three maps display the breakdown of the best fitting template in each pixel according to values of color excess parametrized in magnitudes, metallicity relative to the sun's, and star formation rate in $M_{\odot} / y r$. The redshifts of all pixels have been fixed to that of the galaxy and the "best fitting age" of the galaxy has been computed as described in $\S 4$.

This simple representation of the result of our analysis allows for direct insight into the underlying characteristics of the galaxy. In particular, in all three maps, the morphological details of the galaxy are clearly recognizable. The dark knots seen in the F606W image are also clearly visible in the other maps. The SFR map distinctly displays the arm and inter-arm regions with the former showing a SFR an order of magnitude higher than the latter. A critical value that needs to be associated with this estimate of the SFR per pixel, is the variance in this estimate. As we will show later on, most of the sky pixels have extremely large errors and thus, once the SFR is properly weighted, do not contribute significantly to the overall SFR of the galaxy. These pixels are mostly those at the periphery of the galaxy. This is evident in Figure 4

The obscuration map is perhaps even more dramatic. While generally displaying low color excess, large regions of the galaxy do show considerable amounts of dust. The core of the galaxy, for example, exhibits a $\sim 0.4$ magnitudes of obscuration along the line of sight. The arm regions seem to have pockets of lower obscuration surrounded by regions at higher obscuration and generally appears to be quite patchy, in accordance with observations 
(Trewhella 1998). It is interesting to note that in the obscuration map the knots present in the F606W image are even more evident than in the SFR map where the SFR seems quite uniform across the arm regions.

The metallicity map displays large regions of the galaxy at $Z \sim \frac{1}{50} Z_{\odot}$, but also small pockets in excess of solar. The metallicity map seems to correlate quite well with the SFR map in that all the regions with higher metallicity are indeed those with ongoing star formation as one might expect. As in the SFR map, the outskirts of the galaxy, where the contributions come from mostly sky pixels, exhibit very low metallicities and obscuration, a sign that the sky is generally described by templates with short e-folding times, no extinction and low metallicity.

Thus, by making few simplifying assumptions, we are able to describe a galaxy in terms of its main physical processes at the pixel-by-pixel level and hence discover a previously unseen view. We can envision several scenarios in which this technique might be employed to gain information on the local conditions within galaxies. In particular, as far as the SFR of galaxies is concerned, pixel- $z$ can effectively separate high and low star forming regions in galaxies and, provided a sufficiently large sample of galaxies is available, trace their history. This information, together with a measure of the fraction of old versus young star forming regions, will also provide clues on the clustering properties of star formation. Coupled with information on obscuration and metallicity, pixel- $z$ should be able to return at a minimum direct morphological information and, at best, a new perspective on the evolution of galaxy morphology.

The galaxy in Figure 5 display a substantially different behavior. This galaxy is a face on spiral with clear star formation activity in its spiral arm. The central regions of the galaxy exhibit low star formation activity, solar metallicity and low obscuration. The central region is surrounded by an extended star forming envelope which clearly traces the 
spiral design. Interestingly, the lower right corner of the metallicity map, seems to suggest an earlier burst of star formation in the history of this galaxy. This region is characterized by solar and super-solar metallicity with low obscuration and a moderate ongoing star formation rate.

Clearly, the use of the pixel-z technique can return particularly interesting insights on the link between the morphology of a galaxy and it's physical constituents. We will investigate this link in a forthcoming paper.

\subsection{Effect of photometric redshift uncertainty}

Perhaps an even more important aspect of the pixel-z technique itself is the understanding of the reliability of its estimate. While Figure 4 provides a great deal of immediate information on the local characteristics of a galaxy, this information may at the same time be misleading unless proved reliable.

To do so, we need to consider the source of our uncertainties. First and foremost we need to address the effect the photometric redshift error. To do so, we ran a series of Monte Carlo simulations on the subsample of HDFN galaxies with known spectroscopic redshifts. We added to the redshift of each of these galaxies a randomly distributed Gaussian error with a dispersion ranging from $\sigma_{z}=0.05$ up to $\sigma_{z}=0.2$. We then ran the pixel- $z$ pipeline on these galaxies and recovered the difference in the best fitting template and hence in the underlying physical properties described by the SEDs.

The results are shown in Figure 6. The method is rather robust. Each panel represents

the distribution of deviations from the initial estimate of the properties of galaxies embedded in the SED templates in two photometric redshift error regimes. The solid line represents a marginal error of $\sigma_{z}=0.05$ in redshift (an uncertainty typical of the photometric redshift 
techniques) and produces an extremely narrow peak around the correct value. The dashed line is the result of a much broader redshift error distribution of $\sigma_{z}=0.2$. Even with such a large uncertainty, the number of templates used by pixel-z seem to be able to compensate for the errors. The distribution observed in this case is almost unchanged.

\subsection{Error Maps}

The other source of uncertainty in the pixel-z technique is the intrinsic error associated with the properties of each pixel derived from the best fitting SED. To determine and understand the source and magnitude of this error and how it propagates throughout the pipeline, we need to make use of all the available information.

For each of the pixels in a galaxy, in fact, we have far more information than the best fitting template. Each pixel is characterized by a likelihood function result from the fit, i.e. the value of the likelihood sampled along the 2160 templates. The maximum of this likelihood returns the best fitting SED for a particular pixel, which in turn can be decomposed into four quantities that uniquely determined the SED, namely: age of the stellar population, e-folding time of an exponentially decaying SFR, obscuration and metallicity.

Thus, the likelihood is, in reality, a complex four dimensional function of the main physical quantities that drive the SED. The best fitting template is nothing but the global maximum of this function. Here we are interested in recovering a measure of our uncertainty on each of the four axes of variability of the likelihood function. To do so, we need to be able to "collapse" the four dimensional likelihood function to each one of its dimensions in turn. This can be achieve by calculating the marginalized likelihood function along each axis. 
The results of this procedure for one of the pixels in an HDFN galaxy is shown in Figure 7. The four panels represent marginalized likelihood functions along different axes. The shape of the four functions is quite typical of the behavior of pixels in the HDFN galaxies and serves as a good illustrative example to show how we calculate our uncertainties. While the four dimensional likelihood was sampled at 2160 points corresponding to each of the templates, the likelihoods shown in Figure 7 are one dimensional likelihood functions sampled at the values used to parametrize the SED: age, e-folding time, color excess and metallicity. Thus, the age likelihood function, for example, is sampled at 10 different points corresponding to ages ranging from 0.1 to 15 Gyrs. The other three panels are sampled at 6 different points corresponding to the choices we made in $\S 3$ for obscuration, metallicity and e-folding time.

For one dimensional likelihood functions with one degree of freedom, such as those in Figure 7 , a measure of the $1 \sigma$ uncertainty (which corresponds to a $\Delta \chi^{2}=1$ or a normalized likelihood of $\mathcal{L}=e^{-1 / 2}$ ) can be obtained simply by calculating the range of parameter space intersected by the $1 \sigma$ line. For this particular pixel, for example, while the obscuration and metallicity likelihood functions display a rather sharp peak, and thus produce small uncertainties in these measures, age and e-folding time show much broader likelihood functions which in turn correspond to larger uncertainties.

If we repeat the above exercise for all the pixels in all galaxies of the HDFN, we can associate with each pixel four uncertainties. The results are shown in Figure 8, where the age dependence has been suppressed as we compute the "best fitting age" for the galaxy using the method described in $\S 4$. As expected, those pixels with the highest signal-to-noise ratio in all band-passes, typically pixels well inside the object and commonly referred to as "source pixels", have relatively small errors in contrast with "sky pixels". Direct comparison with the F606W image of Figure 8 underlines how the SFR uncertainty, for example, is 
indeed reliable only within the source and rapidly degrades outward. Recalling the picture

of the SFR we obtained from Figure 4, we see that most of the high SFR at the outskirts of the galaxy, once properly weighted by its uncertainty, carries a small weight in the estimate of the total SFR in the galaxy. Perhaps even more dramatic is the case of the metallicity uncertainty. The dark region represents pixels in the galaxy where the metallicity estimate is reliable to less than 1 dex. These regions, are almost a one-to-one map of the optical image shown. As we move outward the estimate degrades rapidly becoming unreliable with uncertainties greater than 0.5-0.6 dex. A similar result is seen in the color excess error map.

It now becomes clear that, without the aid of error maps such as the ones in Figure 8, any estimate of age, SFR, color excess and metallicity are limited by the unknown reliability. Indeed, much of the computational time spent on the HDFN galaxies by pixel-z was occupied by the calculation of the maximum likelihood of the fit and its proper marginalization. By correctly weighting all the pixels in a galaxy by the appropriate error maps, we can move on to use the information contained in the galaxy maps of Figure 4 and measure the star formation history (hereafter SFH) of galaxies in the HDFN. Before doing so, however we will address the source of uncertainty and degeneracies introduced by the particular SEDs selected for the analysis.

\subsection{SED Degeneracies}

Finally, we would like to address the issue of template degeneracy. Due to the large number of templates used, one might expect a certain degree of degeneracy among the returned physical quantities that determine the single SED of a pixel. To quantify this statement Figure 9 shows the time evolution of obscuration and metallicity. We have taken the best fitting color excess and metallicity, as returned from the best fitting template for each of the pixels in the HDFN galaxies, and fitted a spline surface to examine the combined 
evolution. This approach assumes this relation to be smooth on scales of $\Delta z \sim 0.5$, which is the typical bin size for our star formation history calculations (see the following Section).

At low redshift the extent of this degeneracy is clearly visible. Pixels whose best fitting SED are characterized by solar and above solar metallicities tend to inherently be more obscured than those at lower metallicities. This behavior seems to be rather strong at redshifts below one. As we look at pixels that belong to systems at higher redshift, this behavior seems to change in favor of a flattening of the surface in the extinction direction suggesting that at $z \sim 3$ obscuration is not a good indicator of the underlying metallicity distribution.

At the same time a general steady increase in the metal content of pixels as a function of redshift is evident in Figure 9. This behavior can perhaps be understood in terms of a luminosity selection effect. It is well established that present-day galaxies exhibit a clear trend between B-band luminosity and the oxygen abundance of their H II regions (see Skillman, Kennicutt, \& Hodge 1989). This metallicity-luminosity relation extends across morphological types and over 9 mag in luminosity and appears to hold at least back to $z \sim 0.4$ (Kobulnicky \& Zaritsky 1999). It seem plausible that at high redshift we are more likely (particularly in $U_{300}$-band) to observe luminous star forming galaxies which, in turn, are generally more metal rich (Nagamine et al. 2000; Melbourne \& Salzer 2002). This behavior must be the result of the role mass plays in determining the galaxy formation history, which sets the chemical enrichment history. Mass must regulate either the rate at which elements are produced by star formation or the ease with which they can escape the gravitational potential of the galaxy (or both).

Degeneracies, particularly with a very large number of templates which describe a wide range of underlying physical characteristics of the stellar population, are to be expected. It is important, however, to be aware of the interplay among the different physical parameters 
that regulate the underlying SED in each pixel. To first order Figure 9 demonstrates that these degeneracies can induce strong correlations among the physical quantities at play. The information we gain by examining Figure 9 can now be used to interpret both the galaxy and error maps of Figures 4, 5 and 8.

Finally, it is also important to underscore that the major source of uncertainty might indeed be the SED models themselves. In fact, some of the underlying spectral synthesis models we used might be inaccurate to describe the properties of stellar populations or in the worst case completely wrong. Spectra synthesis models for solar metallicity stellar populations are now pretty well defined for optical photometry, but such models are very much based on extrapolations for very sub-solar and super-solar populations, and remain relatively untested in the $\mathrm{UV}$.

\section{Star Formation History}

Using the framework of the previous two sections, we compute the SFR of all pixels in all galaxies in the HDFN. At this stage of our analysis we have information not only on the SFR of individual galaxies as a whole, but, perhaps as importantly, on the SFR for each of the pixels in the galaxy. As we shall see this information can be used to provide a different view of the star formation history of the universe.

The galaxies in the HDFN span a range in luminosities and look-back times. As a result, in order to be able to convert the star formation histories of individual galaxies (and their pixels) into a comoving average, we need to weight each galaxy (or pixel) by $1 / V_{\max }$ (Schmidt 1968; Bouwens et al. 1998). $V_{\max }$ represents the maximum volume within which

a galaxy of a given apparent magnitude $m_{U}$ and redshift $z_{\text {gal }}$ could still have been observed 
in the HDFN:

$$
V_{\max } \equiv \int_{\Omega} \int_{0}^{z_{\max }} \frac{\mathrm{d}^{2} V}{\mathrm{~d} \Omega \mathrm{d} z} \mathrm{~d} z \mathrm{~d} \Omega
$$

where $\Omega$ is the solid angle subtended by the HDFN, and $z_{\max }$ is the upper redshift limit of detectability for a galaxy with absolute magnitude

$$
M_{U}=m_{U}-5 \log d_{\mathrm{L}}(z)-25-K_{U}(z),
$$

at a luminosity distance $d_{\mathrm{L}}(z)$ with a $U_{300}$-band K-correction $K_{U}(z)$.

Since $z_{\max }$ depends on the galaxy SEDs, we must account for the $K$-correction when calculating $V_{\max }$. The procedure adopted to compute $V_{\max }$ for both galaxies and pixels is identical, however, the SED of each galaxy and of the pixels that belong to it, differ considerably and so will their K-corrections. The photometric redshift of each galaxy in the sample, $z_{\text {gal }}$ is derived by using the maximum likelihood approach of equation (1), where the small set of CWW templates is used to solve for both redshift and SED. By contrast, for each of the pixels in the galaxy, the redshift is held fixed at $z_{\text {gal }}$, while equation (2) is maximized for the best fitting SED selected from the 2160 templates we generate using the Bruzual and Charlot spectrophotometric synthesis models (Bruzual \& Charlot 2000). For each of these pixels, the best fitting SED is used to compute the appropriate redshift dependent $\mathrm{K}$-correction in the $U_{300}$-band. This procedure requires equation 5 to be solved iteratively in order to solve for $z_{\max }$.

Figure 10 shows the star formation history of galaxies and their pixels in the HDFN. The crosses represent the individual galaxy contribution to the star formation history of the universe as determined by its $U_{300}$-band luminosity converted into star formation rate according to equation (3). Below each galaxy, we show as points the contribution to the star formation history of the universe of the individual pixels within each galaxy. The comoving averaged contribution of all pixels in redshift intervals is shown as filled circles. By comparison, we show as open squares a measurements of the UV star formation history 
taken from several sources in the literature.

We find the geometric mean of the star formation rate in the HDFN to be $S F R \sim 0.19 \pm 0.22 \mathrm{M}_{\odot} \mathrm{yr}^{-1} \mathrm{Mpc}^{-3}$. The large dispersion is imputable mainly to a few galaxies with SFR well above $10 \mathrm{M}_{\odot} \mathrm{yr}^{-1} \mathrm{Mpc}^{-3}$, while the bulk of the population remains well below this value.

Immediately below each galaxy, we also show as points the contribution to the star formation history of the universe of the individual pixels within each galaxy. Typically the majority of the objects in the HDFN are rather small, being just a few tens of pixels, but there are several large galaxies containing a few thousand pixels. The mean SFR per pixel in the $\operatorname{HDFN}\langle S F R\rangle \sim 0.004 \pm 0.052 \mathrm{M}_{\odot} / \mathrm{yr} / \mathrm{Mpc}^{3}$ is also characterized by small star formation rates with a large dispersion driven by the clumps of star formation present in large galaxies such as the one shown in Figure 4.

The comoving averaged contribution of all pixels in redshift intervals is then calculated and is shown as filled circles. By comparison, we show as open squares a measurements of the UV star formation history taken from several sources in the literature (Gallego et al. 1995; Lilly et al. 1995; Madau et al. 1996). Our estimates generally agree with the current picture of the star formation history that has emerged in the last few years (Lilly et al. 1995; Cowie et al. 1996; Ellis et al. 1996; Glazebrook et al. 1995; Yee, Ellingson, \& Carlberg 1996): the SFR is, on average, a smooth function of redshift with a sharp increase up to $z \sim 2$ and a decreasing trend at higher redshift. We will show in the next section that this decline is directly related to the poor sampling of the galaxy luminosity function at high redshifts. Once appropriate luminosity functions are derived, the star formation history is much flatter at $z>4$. This behavior is consistent with an exponential decay of the SFR with time. In turn it also underscores that the bulk of stars have formed at redshift higher than $z \sim 2$ (Madau et al. 1998). 
For consistency, we compare our results with other estimates not corrected for obscuration. Despite the lack of an obscuration correction, the result of using the luminosity functions below still supports a flat trend beyond $z>2$.

\subsection{Luminosity Function Correction}

The galaxy luminosity function (LF) is one of the fundamental quantities in observational cosmology. It provides us with a tool to investigate the properties of the galaxy population as a whole. In the context of this work, we are interested in determining how our knowledge, or lack thereof, of the detailed form of the LF as a function of redshift, affects the derived star formation history of the galaxies in the HDF.

In particular, we want to be able to compensate for the fact that at high redshifts we are sampling only the bright end of the galaxy luminosity function and we are therefore systematically underestimating the comoving density of star formation. As a result, somewhat different absolute magnitude ranges are sampled at different redshifts and any smooth functional form used to describe the LF will inevitably return a redshift dependent description of the LF in its parameters.

Nevertheless, a simple functional form, such as that introduced by Schechter (1976)

$$
\phi(M)=(0.4 \ln 10) \phi_{\star}\left[10^{0.4\left(M_{\star}-M\right)}\right]^{1+\alpha} \exp \left[-10^{0.4\left(M_{\star}-M\right)}\right],
$$

where $M_{\star}$ is the characteristic magnitude, $\alpha$ the faint-end slope and $\phi_{\star}$ the normalization factor, will allow an estimate of the total SFR to be derived. We now use equation (6) to estimate the LF within each of the redshift bins of Figure 10. We would like to emphasize here that we are not seeking to trace the evolution of the Schechter parameters with redshift. Instead, we can simply integrate the Schechter LF to a magnitude fainter than our current $U_{300}$-band limiting magnitude, to derive a more complete estimate of the luminosity 
density than available from the detected galaxies alone. Equation (3) will then allow us to convert this luminosity density to a measure of SFR density, as we have done for both galaxies and pixels.

We compute the LF using the parametric maximum-likelihood method of Sandage, Tammann, and Yahil (1979, hereafter STY, see also Lin et al. 1996). The STY method is unbiased by density inhomogeneities in the galaxy distribution and assumes a parametric model for the galaxy LF. In our case, we take as our model for the galaxy LF the Schechter function given in equation (6) and solve for $M_{\star}$ and $\alpha$. The STY method does not return $\phi_{\star}$, the LF normalization constant. To calculate $\phi_{\star}$, we used the minimum-variance estimator of Davis and Huchra (1982). Table 1 lists the best fitting parameters of a Schechter LF in each redshift bin we consider. At high redshift, the small number of (bright) galaxies constrains the fit at the faint end of the luminosity function. The value of $\alpha$ returned by our maximum likelihood estimate there was unphysical, i.e. $\alpha<-2$. To overcome this problem, the LF for galaxies at $z>3.5$ was determined by assuming a conservative value of $\alpha=-1.1$.

The luminosity derived SFR density estimates are shown in Figure 10 as filled squares and the last column of Table 1 contains the fraction of missing comoving density of star formation. We have chosen a limiting magnitude of $U_{300, l i m}=28.5$ corresponding to a $1 \sigma$ detection threshold for point sources in the HDFN (Conti et al. 1999). At high redshift, the fraction of the total luminosity in the detected galaxies decreases substantially compared to that at lower redshifts. It appears evident that for $z<2$ we miss almost half of the star formation and by $\sim 3$, we reach $90 \%$. Above this redshift, if our estimate of the luminosity function is to be trusted, we miss virtually all the SFR.

The picture of the star formation rate that emerges from the luminosity function 
derivation is that of an exponentially increasing comoving density of star formation:

$$
\log \dot{\rho}_{*} \propto(1+z)^{0.12 \pm 0.02}
$$

This result is in agreement with the findings of Lanzetta et al. (2002). In fact, current measurements miss a dominant fraction of the ultraviolet luminosity density and hence of the star formation rate of the universe. According to our results, the comoving density of star formation rate determined from the UV luminosity density of sources in the HDFN, increases monotonically with redshift out to at least redshift of 5 . This behavior can plausibly be explained either by a direct increase of the UV luminosity density with redshift or by an increase in the number of star forming regions as a function of redshift.

To address this issue, we compute the fraction of the UV flux (and hence of the star formation rate) needed to recover $90 \%$ of the detected star formation rate at each redshift bin in Figure 10. We divide the sample of HDFN galaxies (and their pixels) in two broad sub-samples based on their rest frame $(\mathrm{B}-\mathrm{V})$ color: bluer and redder than $(\mathrm{B}-\mathrm{V})=0.32$ respectively. This simple scheme is obviously not intended to be a rigorous separation of a blue versus a red populations of galaxies (or pixels) in the HDFN. We are interested in understanding the fraction of the total number of galaxies and pixels needed to recover a given SFR as a function of redshift. We argue that this quantity must be related to the history of formation of the HDFN galaxies and to their current star formation activity and perhaps linked to a galaxy's morphology.

Figure 11 shows the fraction of star formation rate needed to recover $90 \%$ of the total UV flux in the red and blue population for galaxies (bottom panel) and pixels (top panel). The solid line represents the red population, the dashed line the blue one. The functional dependence with redshift has been smoothed on scales of $z \sim 0.5$ in accordance with the typical width of the redshift bin in Figure 10.

The red population (solid line) at the bottom of Figure 11 represents the evolution of 
the fraction of galaxies needed at any given redshift to recover $90 \%$ of the SFR. For $z>0.5$, this evolution seems to be rather similar to that of the blue population (dashed line). This behavior suggests that up to relatively high redshifts $(z \sim 3)$ a comparable fraction of red and blue galaxies are needed to give rise to the observed SFR. Below a redshift of $z<0.5$ the lack of a suitable blue population (indicated by the size of the statistical error bar) suggests that there are simply not enough low-redshift blue galaxies in the HDF to draw firm conclusions.

The top panel describes a quantitatively different behavior. The solid and dashed lines represent the fraction of pixels in the red and blue galaxies that accounts for $90 \%$ of the SFR. At redshifts $z<1$ we need approximately $20 \%$ of the pixels in red galaxies to account for their SFR. As we move to higher redshifts, we observe a steady increase in the number of pixels required to account for the UV flux out to $z \sim 3$. At higher redshifts the number of galaxies is too small to draw further conclusions (see Figure 10). This increase is a direct consequence of the surface brightness dimming of the host galaxies that harbor these pixels. This claim is supported by a simple exercise that makes use of our ability to use the pixels' SED to coherently move all the pixels in a galaxy to a new redshift taking into proper account the surface brightness dimming due to the expansion of the universe.

First we select a red, bright galaxy at low redshift. We then step in redshift out to $z \sim 3$ in intervals of $\Delta z=0.2$. At each interval we use the SED of each pixel to compute the redshifted SED flux convolved with the HST F300W U-passband filter. We then generate a postage stamp image of the original galaxy and lay it on a grid of pixels with the same resolution of our HDFN images (i.e. $0.08^{\prime \prime} /$ pixel). At each step we properly take into account the new angular size of the galaxy by resampling the flux to match the new size. Resampling is done using a 2D-spline fit to the flux at each redshift. At each redshift, we also dim the flux to account for surface brightness dimming. In this exercise we did not 
take into account the intrinsic luminosity evolution of the galaxy. We were only interested in measuring the effects of surface brightness dimming on the pixels.

This procedure returns the open squares in the top panel of Figure 11. The galaxy seems to follow the general trend observed for the red pixels and hence its behavior is primarily dictated by surface brightness effects. The typical statistical uncertainty associated with each square is indicated in the corner of the figure.

The behavior of the blue galaxies is qualitatively different. The fraction of blue pixels needed to recover $90 \%$ of the flux sharply increases to $\sim 30 \%$ by redshift one and it remains almost unchanged out to redshift $z \sim 3$. We can make use of our direct knowledge of the SED in each pixel to artificially redshift a single galaxy to high redshift and observe its behavior. The results are shown in Figure 11 as open triangles. Surface brightness dimming seems to be able to also explain the observed trend. It appears that while the same fraction of galaxies are contributing to the SFR up to $z \sim 3$, the number of pixels contributing to the flux differs. This difference must be controlled by the relative distribution of pixels within a galaxy as a function of redshift, and ultimately be related to the morphological characteristics of galaxies.

At high redshift, mainly because of surface brightness dimming, we need a similar fraction of blue and red pixels to recover most of the star formation rate. By contrast, at low redshift the two populations of pixels differ in their behavior suggesting that the pixel-z technique can be used to probe this difference. In fact, the blue pixel curve (top panel - dashed line), must hold some insight to the intrinsic distribution of SFR in a galaxy. Where the star formation rate is mostly concentrated at the center of a galaxy, as in the surface brightness dimming induced case of high redshift galaxies, we need roughly the same number of red and blue pixels to explain $90 \%$ of the SFR. However, a much smaller number of pixels is needed at lower redshift to explain the red pixel behavior. This underscores the 
different morphological characteristics of the host galaxies and suggests that we are in the presence of more extended areas of star formation.

Ultimately, this last statement has to be closely connected with the morphological characteristics of a galaxy. Clearly, the information contained in the pixels and extracted using the pixel-z technique, can be used as a probe of these morphological differences. We plan to address the pixel-morphology connection in a forthcoming paper.

Finally, we can make use of our estimate of metallicity to compute the comoving metallicity density of the universe. Our derived luminosity densities were converted to metal enrichment rates to compare with the predictions of Pei \& Fall (1995) and Pei \& Fall (1999) and with the $z>2$ data of Madau et al. (1996) (which, as noted, are based on $1500 \AA$ luminosity densities). The conversion from $\mathrm{L}(2800 \AA)$ to metal enrichment rate is $2.2 \times 10^{-23} \mathrm{M}_{\odot} \mathrm{yr}^{-1} \mathrm{~W}^{-1} \mathrm{~Hz}$. This value differs from the that adopted by Madau et al. (1996) by a factor of approximately 1.6 due, in part, to changes in the stellar synthesis models of Bruzual \& Charlot (2000). Figure 12 shows our results. Solid circles represent our direct estimate, while solid squares represent our integrated luminosity function results.

Our uncorrected estimates are in good agreement with the literature (Lilly et al, 1996 open triangle, Gallego et al., 1995 open circles, Connolly et al., 1997 solid triangles) and with the theoretical models of Pei \& Fall (1999) based on the evolution of the HI content in damped Lyman- $\alpha$ systems. The luminosity function correction shows a remarkable difference as a function of redshift from its uncorrected values, in agreement the findings of this work and with current measurements of the ultraviolet luminosity density (Lanzetta et al. 2002). 


\section{Conclusions and Future Applications}

We analyze the photometric information contained in individual pixels of galaxies in the Hubble Deep Field North (HDFN) using a new technique, pixel-z, that combines predictions of evolutionary synthesis models with photometric redshift template fitting. Each spectral energy distribution template is a result of modeling of the detailed physical processes affecting gas properties and star formation efficiency. The criteria chosen to generate the SED templates is that of sampling a wide range of physical characteristics such as age, star formation rate, obscuration and metallicity. By making use of a marginalized likelihood error analysis were are also able to generate error maps that define the reliability of the template fitting on pixel scales and allow for the separation of the interplay among dust, metallicity and star formation histories.

Our technique has the clear advantage of being able to probe the star formation history of the universe independently of morphological transformations. In fact, the curves shown in the top panel of Figure 11 record the star formation activity of stars in galaxies without any prior knowledge of their morphological distribution. At the same time, however, pixel-z can be used to constrain the distribution of star forming regions within galaxies in different redshift ranges. This "star formation clustering" has to be closely connected with the formation history and morphological characteristics of a galaxy. Figure 11 strongly implies that the pixel-z approach to studying the evolution of star formation in galaxies returns a new description of the role SFR plays in determining the morphological characteristics of system. This results suggests that pixel-z does indeed provide us with new insights on galaxies.

Moreover, thanks to the large number of templates used and the range of physical quantities they sample, pixel-z is able to return a detailed snapshot of a system. Clear examples of this are shown in Figures 4 and 5 where two different galaxies are examined. 
A key feature of pixel-z is its ability to make use of the physical quantities recovered to examine the degeneracies in the SED templates and the role they play in our understanding of individual systems. Dust content, metallicity and star formation history alter the colors of galaxies in ways which are by no means orthogonal (Kodama, Bell, and Bower 1999; Thompson et al. 1999,see)). Figure 9, for example, underscored the importance of understanding the evolution of the metallicity-obscuration relation in order to be able to interpret the characteristics of an individual galaxies. Its important to mention that these conclusions depend on the universal validity of the Calzetti (1997) extinction law.

Finally, the integrated light from an entire galaxy or from individual pixels represents an average over cosmic time of the stochastic star formation episodes of individual galaxies, and will follow a relatively simple dependence on redshift. By examining the evolution of the observed UV flux, which in turn is proportional to SFR, we hope to gain valuable insights on the mechanisms which may prevent the gas within virialized dark matter halos from radiatively cooling and turning into stars at early times, or on the epoch when galaxies exhausted their reservoirs of cold gas (Madau et al. 1996).

However, it is apparent by inspection of Table 1 how at high redshifts we are progressively sampling only the bright part of the galaxy luminosity function. Hence, we compute an estimate of the possible uncertainty in the global star formation rate induced by an incomplete luminosity function by asking what portion of the total luminosity we are missing. Figure 10 shows the luminosity function corrected star formation rates and indicates that these corrections are indeed quite strong. Our results show that the comoving density of star formation rate, determined from the UV luminosity density of sources in the HDFN, increases monotonically with redshift out to at least redshift of 5 . This behavior can plausibly be explained by a smooth increase of the UV luminosity density with redshift coupled with an increase in the number of star forming regions as a function of redshift. 
Furthermore, we find the overall metal enrichment rate history to be consistent with the predictions of Pei \& Fall (1999) based on the evolving HI content of Lyman- $\alpha$ QSO absorption line systems.

With the development of wider and more efficient space based cameras, such as the ACS, deep, multi-band surveys will become the new testing grounds for the pixel-z technique. At present pixel-z seems to be the best available tool to help shed some light on the nature of star formation at moderate to high redshift and its connection to galaxy formation processes.

We wish to thank Jeff Gardner for helpful discussions and Michael Fall for providing his metal enrichment data in electronic form. We acknowledge the referee Roberto Abraham for comments and suggestions which helped to improve the overall clarity of the manuscript. AMH acknowledges support provided by the National Aeronautics and Space Administration (NASA) through Hubble Fellowship grant HST-HF-01140.01-A awarded by the Space Telescope Science Institute (STScI). STScI is operated by the Association of Universities for Research in Astronomy, Inc., under NASA contract NAS5-26555. This work has been supported by NSF Career grant AST99-84924, by NASA Applied Information Systems Research grant NAG 5-9399 and by NASA LTSA Grant NAG5-8546. 


\section{REFERENCES}

Abraham, R. G., Ellis, R. S., Fabian, A. C., Tanvir, N. R., \& Glazebrook, K. 1999, MNRAS, 303,641

Bolzonella, M., Miralles, J.M., Pelló, 2000 A\&A, 363, 476

Bruzual, Charlot, S. 2000, in preparation

Budavári, T., 2000, Szalay, A.S., Connolly, A.J., Csabai, I., Dickinson, M. 2000, AJ, 120, 1588

Budavári, et al. 2001, AJ, 122, 1163

Budavári, T., et al., 2003, in preparation

Calzetti, D. Armus, L., Bohlin, R.C., Kinney, A.L., Koornneef, J., Storchi-Bergmann, T. 2000, ApJ, 533, 682

Coleman, G.D., Wu, C.C. \& Weedman, D.W. 1980 ApJS, 43, 393

Connolly, A.J., Szalay, A. S., Bershady, M.A., Kinney, A. L. \& Calzetti, D. 1995, AJ, 110, 1071

Connolly, A.J., Csabai, I., Szalay, A.S., Koo, D.C., Kron, R.C., \& Munn, J.A. 1995, AJ, 110,2655

Connolly, A.J., Szalay, A.S., Dickinson, M., SubbaRao, M.U., \& Brunner, R.J. 1997, ApJ, 486, L11

Conti, A., Kennefick, J.D., Martini, P. \& Osmer, P.O. 1999, AJ, 117, 645

Cowie, L. L. 1988, in The Post-Recombination Universe, eds. N. Kaiser \& A. N. Lasenby (Dordrecht: Kluwer), 1 
Cowie, L. L., Songaila, A., Hu, E. M., \& Cohen, J. G. 1996, AJ, 112, 839

Davis, M., \& Huchra, J. P. 1982, ApJ, 254, 437

Dickinson et al. 2003, in preparation

Ellis, R. S., Colless, M., Broadhurst, T., Heyl, J., \& Glazebrook, K. 1996, MNRAS, 280, 235

Fernández-Soto Alberto, Lanzetta, Kenneth M., Chen, Hsiao-Wen, Pascarelle, Sebastian M., Yahata, Noriaki, 2001, ApJS, 135, 41

Fukugita, M., Hogan, C. J., \& Peebles, P. J. E. 1996, Nature, 381, 489

Furusawa, Hisanori, Shimasaku, Kazuhiro, Doi, Mamoru, Okamura, Sadanori ApJ, 534, 624

Gallego, J., Zamorano, J., Aragon-Salamanca, A. \& Rego, M., 1995, ApJ, 455, L1

Glazebrook, K., Peacock, J. A., Miller, L., \& Collins, C. A. 1995b, MNRAS, 275, 169

Gwyn, S. D. J., \& Hartwick, F. D. A. 1996, ApJ, 468, L77

Hopkins, A. M., Connolly, A.J., Haarsma, D.B., Cram, L.E. 2001, AJ, 122, 288

Hogg, D. et al. 1998 AJ, 115, 1418

Kennicutt, R. C., 1998, ARA\&A, 36, 189

Kobulnicky, H. A., \& Zaritsky, D. 1999, ApJ, 511, 118

Kodama, T., Bell, E. F., \& Bower, R. G. 1999, ASP Conf. Ser. 191, Photometric Redshifts and High Redshift Galaxies, ed. R. J. Weymann, L.J. Storrie-Lombardi, M. Sawicki, \& R. J. Brunner, (San Francisco ASP), 160

Lanzetta, K. M., Yahil, A., \& Fernández-Soto, A. 1996, Nature, 381, 759

Lanzetta, K.M., Yahata, N., Pascarelle, S., Chen, H. \& Fernández-Soto 2002, ApJ, 570 
Le Borgne, D. \& Rocca-Volmerange, B. 2002, A\&A, 386, 446

Lilly, S. J., Le Fèvre, O., Hammer, F., \& Crampton, D. 1996, ApJ, 460, L1

Lilly, S. J., Tresse L., Hammer, F., Crampton, D., \& Le Fèvre, O. 1995, ApJ, 455, 108

Lin H., Kirshner, P.R., Shectman, S.A., Landy, S.D., Oemler, A., Tucker, D.L. \& Schechter, P. 1996, ApJ, 464, 60

Lin, H., Yee, H. K. C., Carlberg, R. G., \& Ellingson, E. 1997, ApJ, 475, 494

Loh, E. D., \& Spillar, E. J. 1986, ApJ, 303, 154

Madau, P. 1995, ApJ, 441, 18

Madau, P., Ferguson, H.C., Dickinson, M.E., Giavalisco, M., Steidel, C.C., Fruchter, A. MNRAS, 283, 1388

Madau, Piero, Pozzetti, Lucia, Dickinson, Mark 1998, ApJ, 498, 106

Melbourne, J. \& Salzer, J.J. 2002, AJ, 123, 2302

Nagamine, Kentaro, Cen, R., Ostriker, J.P. 2000, ApJ, 541, 25N

Mobasher, B., Rowan-Robinson, M., Georgakakis, A., \& Eaton, N. 1996, MNRAS, 282, 7

Pei, Y.C. \& Fall S.M. 1995, ApJ, 454, 69

Pei, Y.C. \& Fall S.M. 1999, ApJ, 522, 604

Richards, Gordon T. et al. 2001, AJ, 122, 1151

Sandage, A., Tammann, G.A., \& Yahil, A. 1979 ApJ, 232, 352

Sawicki, M.J., Lin, H. \& Yee, H.K.C., 1996, AJ, 117, 1 
Schmidt, S.J., et al. 2003, in preparation

Schechter, P. L. 1976, AJ, 203, 297

Skillman, E. D., Kennicutt, R. C., Jr., \& Hodge, P. 1989, ApJ, 347, 875

Steidel, C.C., Giavalisco, M., Dickinson, M., Adelberger, K. L., 1996, AJ, 112, 352

Thompson, R.I., Weymann, R.J., Storrie-Lombardi, L.J. 2001 ApJ, 546, 694

Thompson, D., Kelly, A. E., Sawicki, M., Soifer, B. T., \& Mathews, K. 1999, ASP Conf. Ser. 191, Photometric Redshifts and High Redshift Galaxies,ed R. J. Weymann, L.J. Storrie-Lombardi, M. Sawicki, \& R. J. Brunner, (San Francisco ASP), 291

Trewhella, M. 1998, MNRAS, 297, 807

William, R. et al. 2000, AJ, 120, 2735

William, R. et al. 1996, AJ, 112, 1335

Yee, H. K. C., Ellingson, E., \& Carlberg, R. G. 1996 ApJS, 102, 269 
Table 1. LUMinOSITY FUnCTION PARAMETERS

\begin{tabular}{cccccc}
\hline \hline Redshift Range & $N_{\text {gal }}$ & $M_{\star}-5 \log h^{a}$ & $\alpha$ & $\phi_{\star}\left(h^{3} M p c^{-3}\right)^{a}$ & Missing Fraction $^{c}$ \\
\hline & & & & & \\
$0.0<z<1.0$ & 355 & $-17.71 \pm 0.09$ & $-0.79 \pm 0.12$ & $0.082 \pm 0.010$ & $69 \%$ \\
$1.0<z<2.0$ & 338 & $-18.46 \pm 0.09$ & $-0.95 \pm 0.11$ & $0.045 \pm 0.002$ & $33 \%$ \\
$2.0<z<3.5$ & 174 & $-19.40 \pm 0.13$ & $-0.94 \pm 0.15$ & $0.035 \pm 0.005$ & $90 \%$ \\
$3.5<z<5.0$ & 34 & $-20.16 \pm 0.29$ & $-1.10^{b} \pm 0.31$ & $0.019 \pm 0.004$ & $98 \%$ \\
\hline
\end{tabular}

${ }^{\mathrm{a}} H_{0}=75 h \mathrm{~km} \mathrm{~s}^{-1} \mathrm{Mpc}^{-3}$. Errors are formal $1 \sigma$ uncertainties.

${ }^{\mathrm{b}}$ Due to the small number of galaxies in this high redshift bin, the faint end of the luminosity function was, at best, poorly constrained. The value of $\alpha$ returned by our maximum likelihood fit was unphysical, i.e. $\alpha<-2$. Hence, in this redshift range alone, the value of $\alpha$ was held fixed.

${ }^{c}$ Fraction of missing comoving density of star formation. In Figure 10 the observed points (filled circles) are affected by the incomplete sampling of the galaxy luminosity at faint magnitudes as a function of redshift. This in turn determines an underestimate of the SFR in each redshift interval. By integrating the galaxy luminosity function in each redshift range out to $U_{300, l i m}=28.5$ and by transforming this luminosity into SFR according to equation (3), we are able to compute the necessary correction to the points in Figure 10 (filled squares). 
Fig. 1. - Distribution of pixels as a function of redshift in a bright, large spiral galaxy in the HDFN. For each pixel a photometric redshift is computed using the template fitting technique. For each of the pixels, the resulting photometric redshift estimate, is simply a function of the flux in each of the available band-passes. When applied to the entire galaxy (i.e. the sum of the contributions from individual pixels), the photometric redshift returned should correlate with the number of pixels that have photometric redshifts near the galaxy overall redshift.

Fig. 2.- Same as Figure 1, but for a small and faint galaxy in the HDFN. The more interesting features of this figure reside in the number and strength of the 3 peaks shown and in the information they might contain. One can envision a scenario in which two objects at different redshifts, but close in angular distance, might be separated by using this method. This appears to be on such case (Conti et al. 1999). See the text for details.

Fig. 3.- Top: Star formation rate computed from aperture photometry for all galaxies in the HDFN versus the star formation rate derived from the sum of all pixels in the same galaxies. The line represents the one-to-one relation and is not a fit to the data. The agreement is quite good, even though the pixel-z result seems to slightly overestimate the overall SFR. This behavior can be understood by noticing that for large galaxies a fixed aperture might not include all pixels. Bottom: Residual uncertainties in the measurement of the star formation rate. For those galaxies with no UV detection, we have assumed an upper limit for their fluxes as determined from the $U_{300}$ rms maps. These objects are generally small and, when using aperture photometry, the aperture is likely to include many background pixels, which in turn give the appearance of larger star formation rates. These outliers are clearly visible in the residual plot. 
Fig. 4.- Result of the fit of the 2160 SED templates to a large spiral galaxy in the HDFN. The top left map shows the galaxy in the F606W WFPC2 filter. The other three maps display the breakdown of the best fitting template in each pixel according to values of color excess parametrized in magnitudes, metallicity relative to the sun's and star formation rate in $M_{\odot} / y r$. The redshifts of all pixels have been fixed to that of the galaxy and the "best fitting age" of the galaxy has been computed as described in $\S 4$. See the text for details.

Fig. 5.- Same as Figure 4 for another galaxy in the HDFN.

Fig. 6.- Estimate of the photometric redshift error propagation in the pixel-z pipeline. Each panel represents the distribution of deviations from the initial estimate of the properties of galaxies embedded in the SED templates in two photometric redshift error regimes. The solid line represents a marginal error of $\sigma_{z}=0.05$ in redshift (an uncertainty typical of the photometric redshift techniques) and produces an extremely narrow peak around the correct value. The dashed line is the result of a much broader redshift error distribution of $\sigma_{z}=0.2$. Not surprisingly the distribution of deviations broadens, but still within very acceptable limits.

Fig. 7.- Marginalized likelihood functions along different axes. While the four dimensional likelihood was sampled at 2160 points corresponding to each of the templates, the likelihoods shown are one dimensional likelihood functions sampled at the values used to parametrize the SED: age, e-folding time, color excess and metallicity. Thus, the age likelihood function, for example, is sampled at 10 different points corresponding to ages ranging from 0.1 to 15 Gyrs. The other three panels are sampled at 6 different points corresponding to the choices we made in $\S 3$ for obscuration, metallicity and e-folding time. The horizontal line represents a $1 \sigma$ uncertainty. The likelihoods shown are the result of a polynomial fit to the data. 
Fig. 8.- Error maps for the galaxy shown in Figure 4. These maps are obtained by computing the marginalized likelihood for each pixel in the galaxy as described in $\S$ 4.4. Note that the SFR is indeed reliable only within the source and rapidly degrades outward. Most of the high SFR at the outskirts of the galaxy, once properly weighted by its uncertainty, carries a small weight in the estimate of the total SFR.

Fig. 9.- Evolution of obscuration and metallicity as a function of redshift for all pixels in the HDF-N. At low redshift pixels whose best fitting SED are characterized by solar and above solar metallicities tend to inherently be more obscured than those at lower metallicities. The nature of this degeneracy is rather strong at redshifts below one. At higher redshifts, this behavior changes in favor of a flattening of the surface in the extinction direction suggesting that at $z \sim 3$ obscuration is not a good indicator of the underlying metallicity distribution. A general steady increase in the metal content of pixels as a function of redshift is also evident. This behavior can be understood in terms of a luminosity selection effect whereby higher luminosity systems are also preferentially metal rich.

Fig. 10.- Star formation history of galaxies and their pixels in the HDFN. The crosses represent the individual galaxy contribution to the star formation history of the universe as determined by its $U_{300}$-band luminosity. Below each galaxy, we show as points the contribution to the star formation history of the universe of the individual pixels within each galaxy. The comoving averaged contribution of all pixels in redshift intervals is shown as filled circles. By comparison, we show as open squares a measurements of the UV star formation history taken from several sources in the literature. The luminosity derived SFR density estimates are show as filled squares and the last column of Table 1 contains the fraction of missing comoving density of star formation. 
Fig. 11. - Fraction of the UV flux (and hence of the star formation rate) needed to recover $90 \%$ of the star formation rate in each redshift bin. A blue and a red sample were extracted from the HDFN galaxies based on B-V rest frame colors. The top panel shows the fraction needed to recover $90 \%$ of the total UV flux for HDFN galaxies. The bottom panel looks at pixels. The solid line represents the red population, the dashed line the blue one. The functional dependence with redshift has been smoothed on scales of $z \sim 1$ in accordance with the typical width of the redshift bin in Figure 10.

Fig. 12. - Metal enrichment of the universe rate as a function of redshift. Solid circle represent our direct estimate, while solid squares represent our luminosity function correction. Our uncorrected estimates are in good agreement with the literature (Lilly et al., 1996 open triangle, Gallego et al., 1995 open circles, Connolly et al., 1997 solid triangles) and with the theoretical models of Pei \& Fall (1999) (solid and dashed lines). The luminosity function correction shows a remarkable difference as a function of redshift from its uncorrected values. See the text for details. 


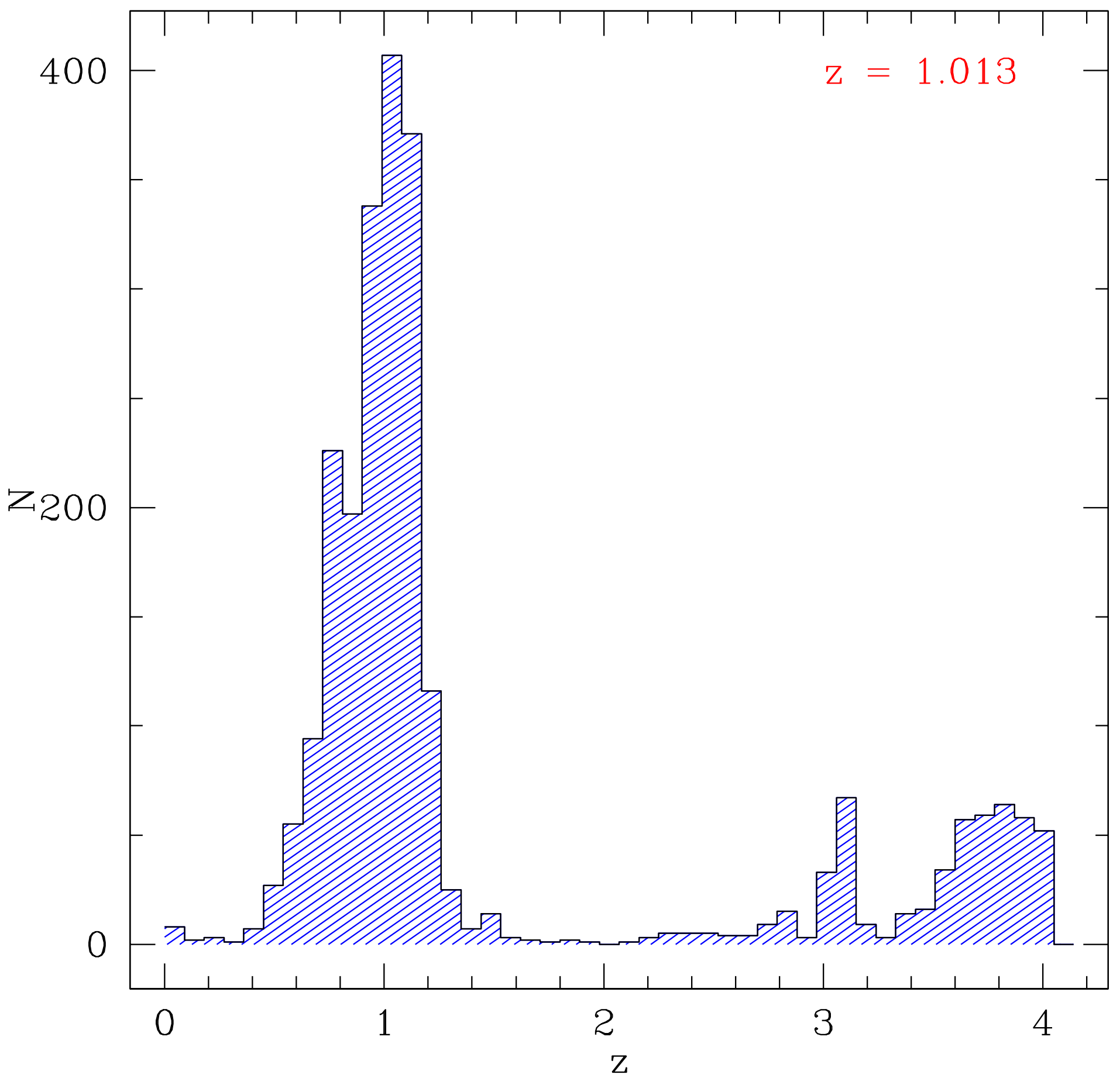




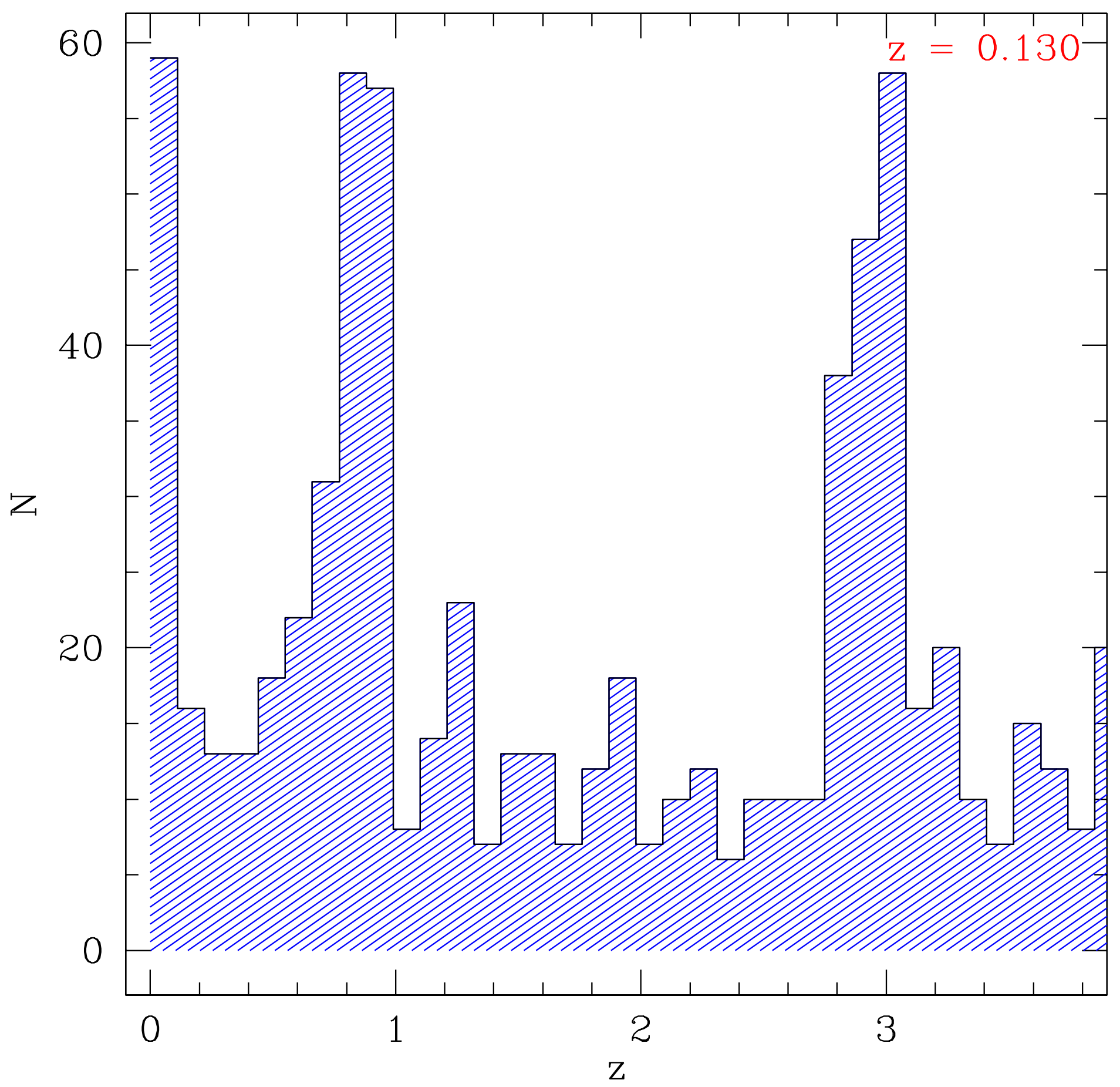




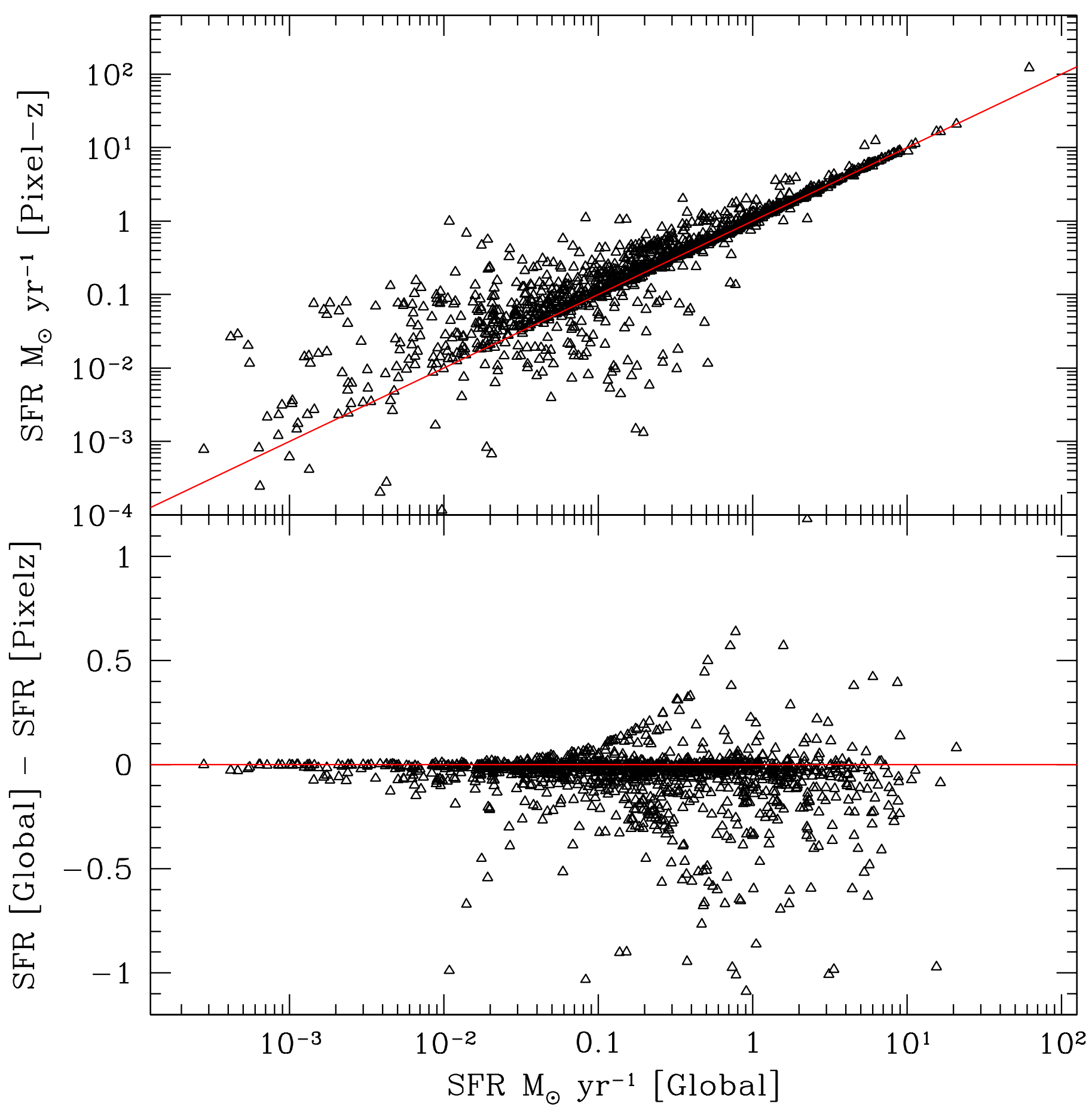



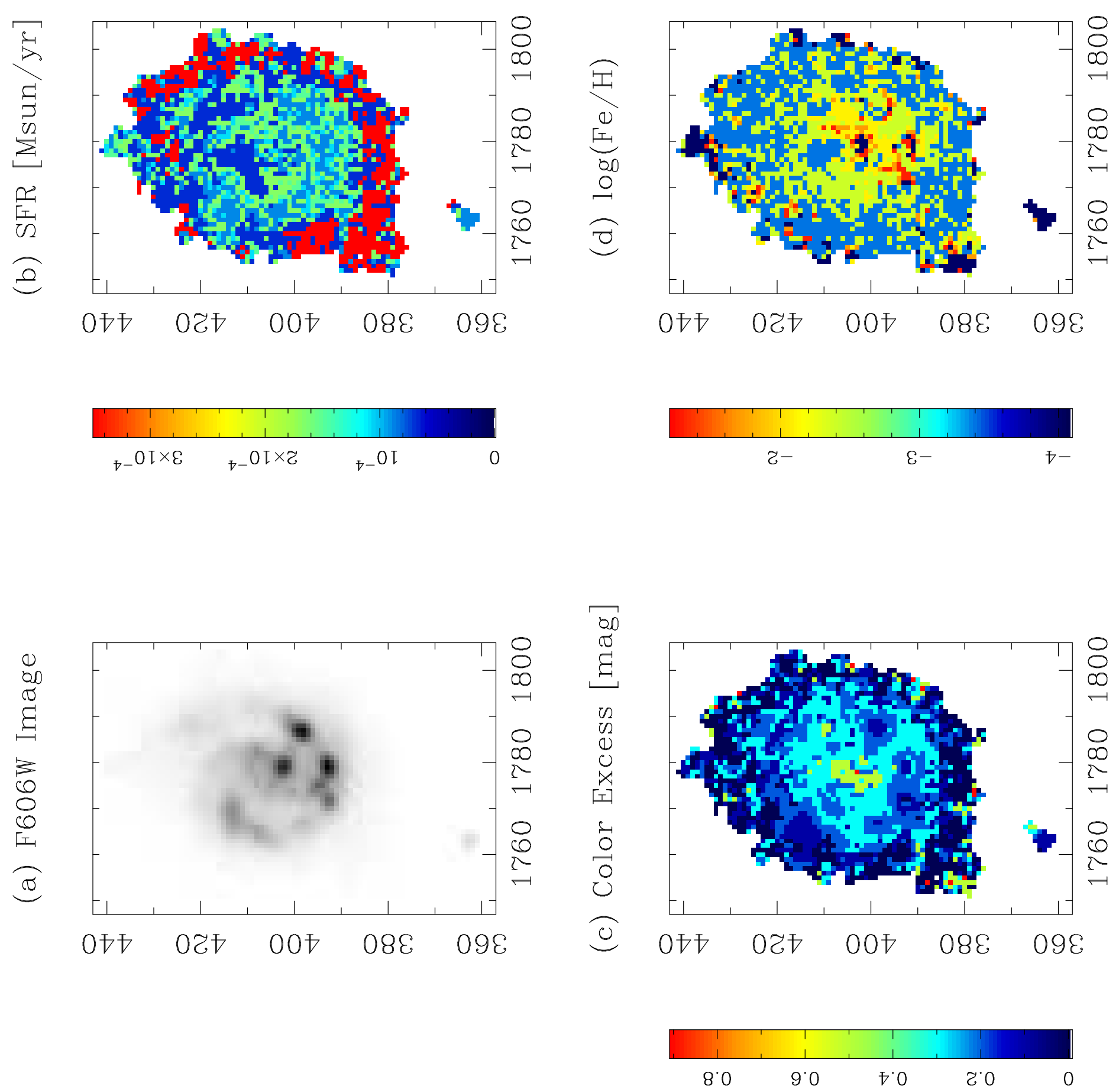

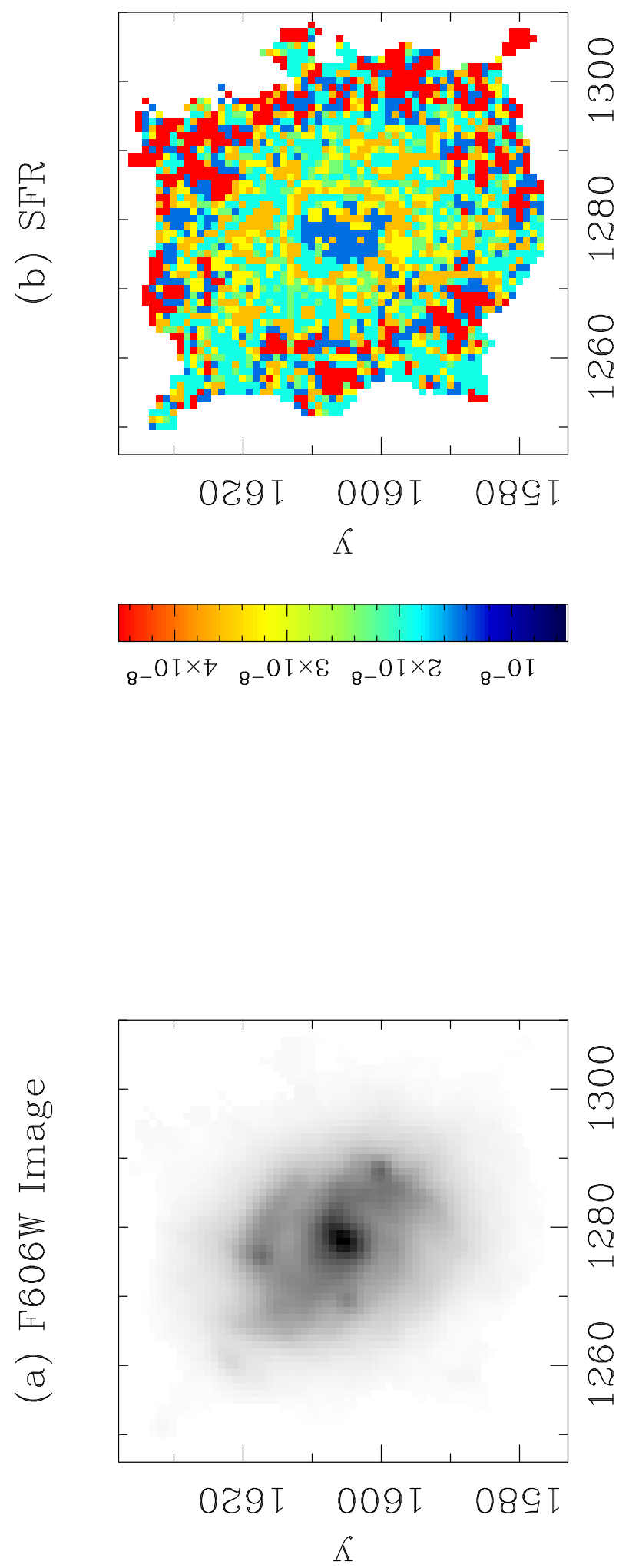
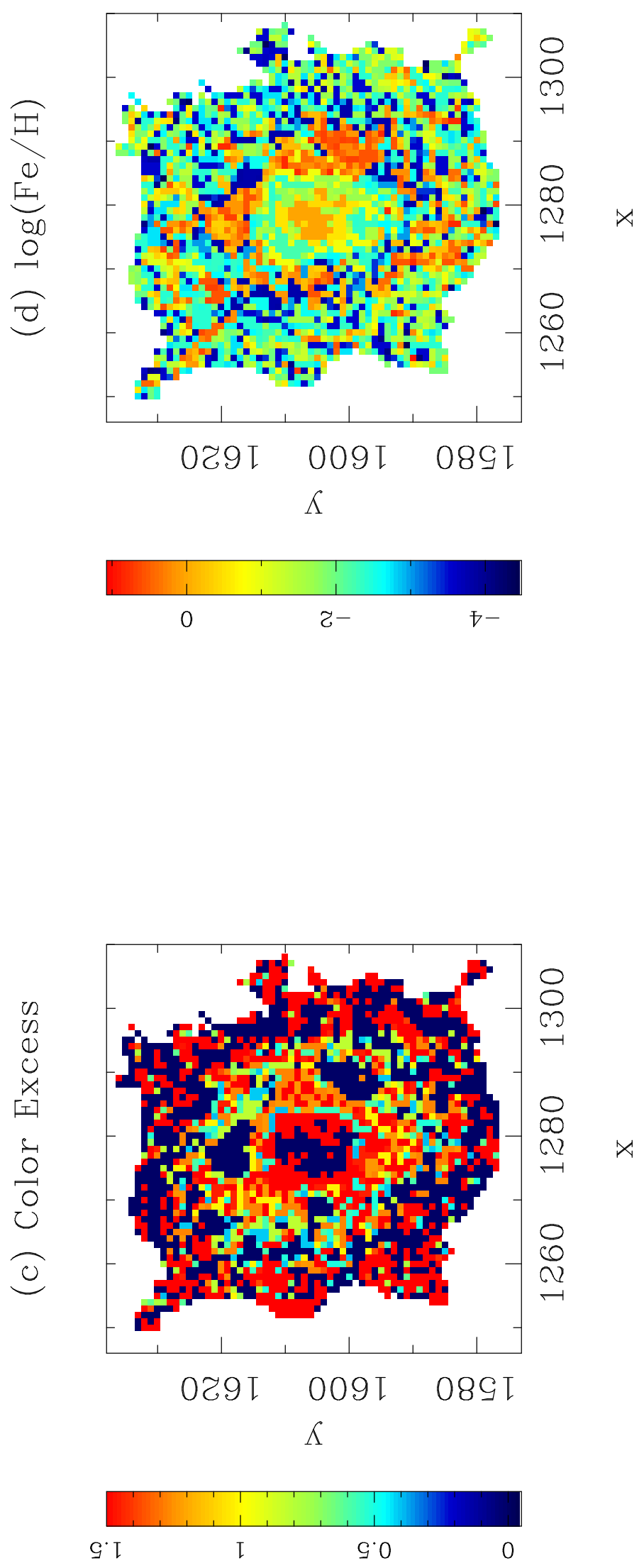

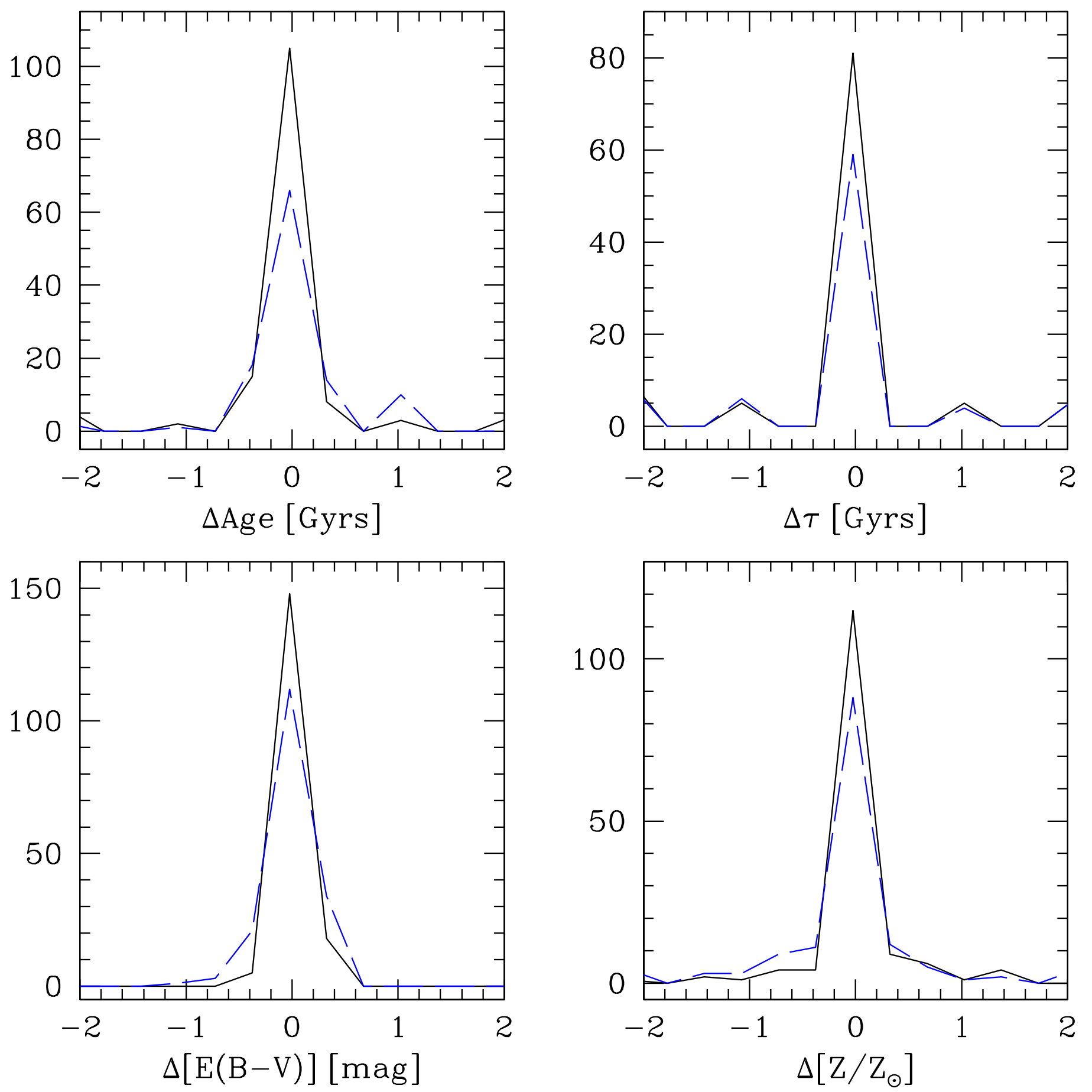

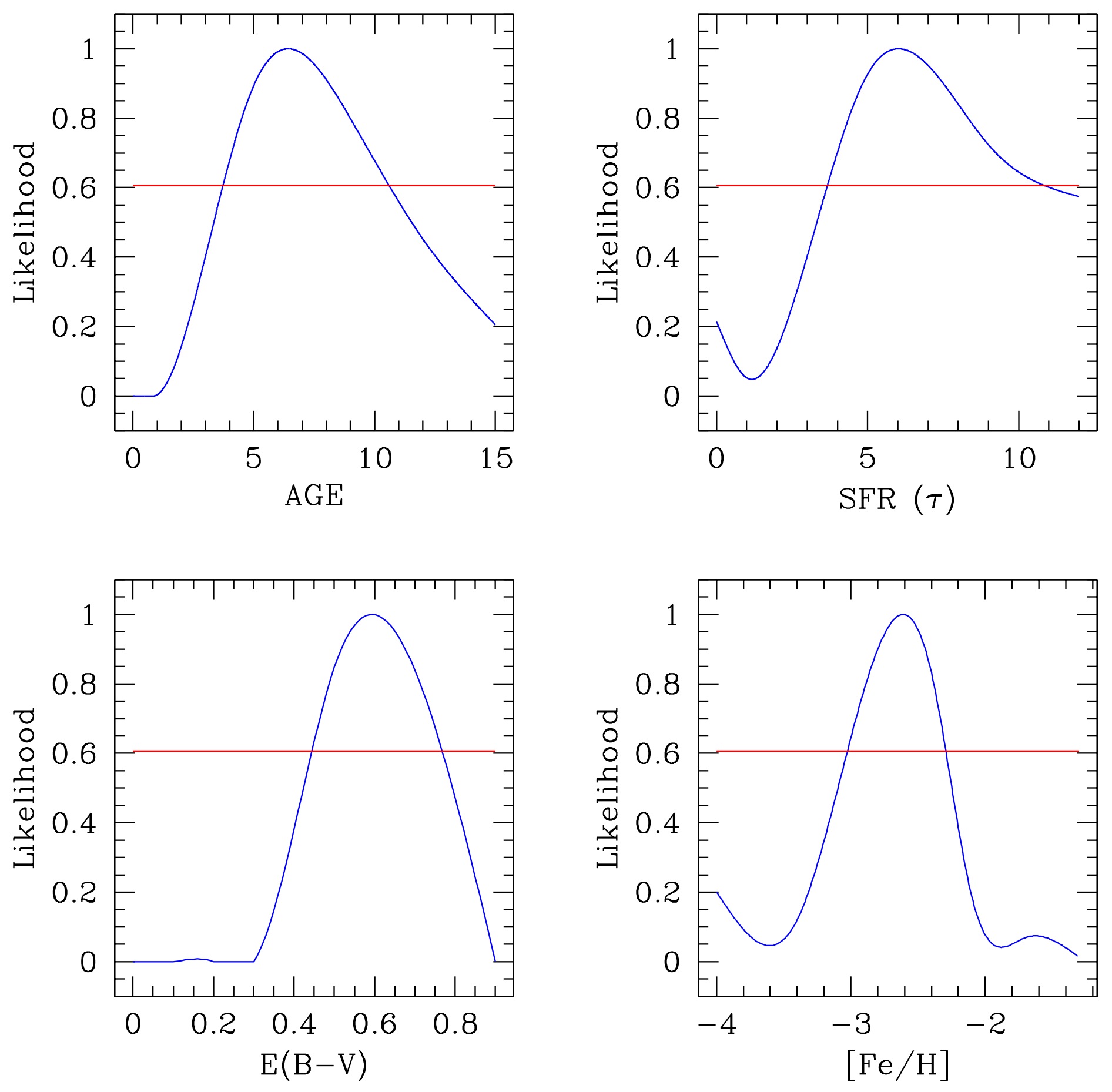

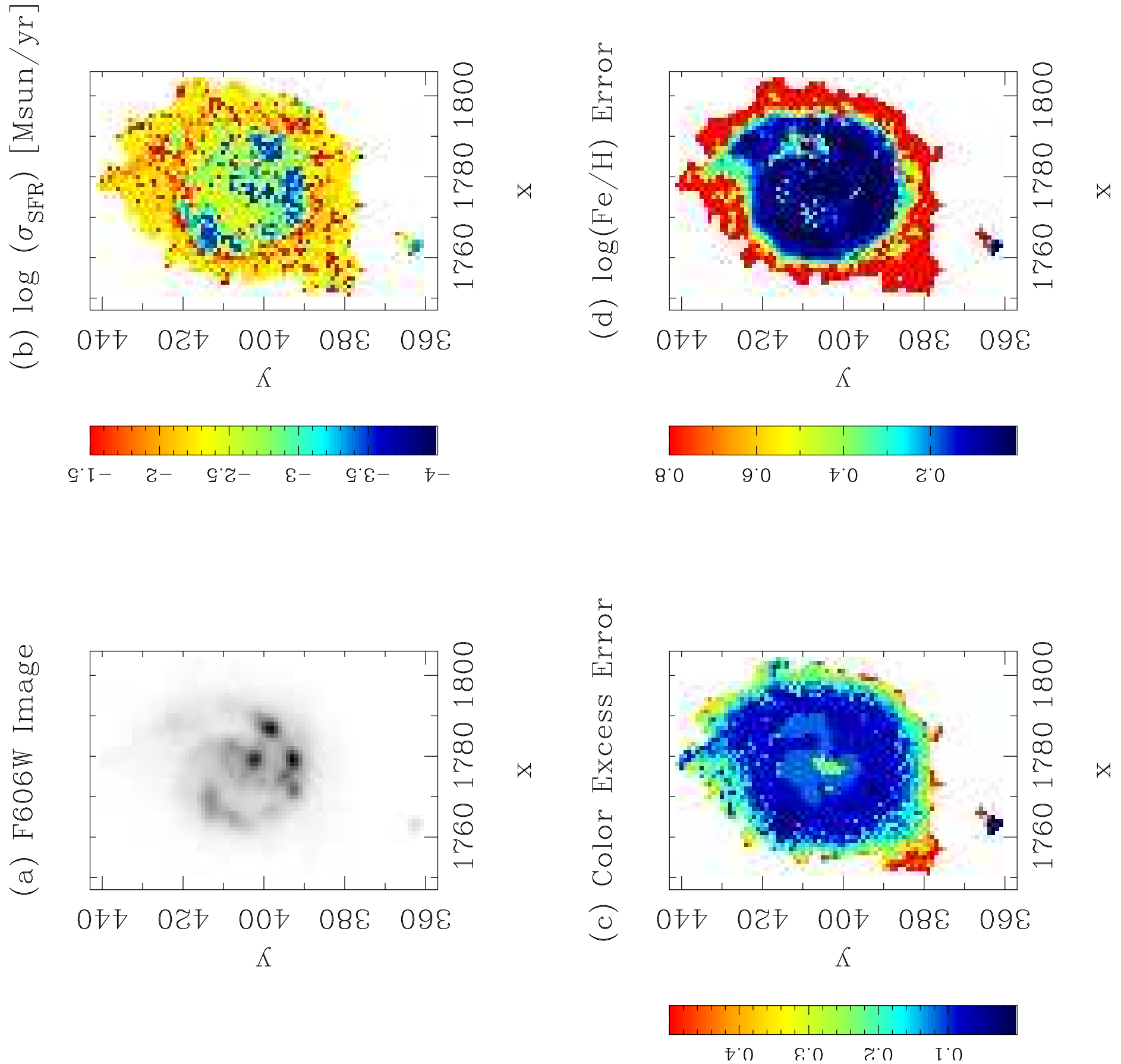


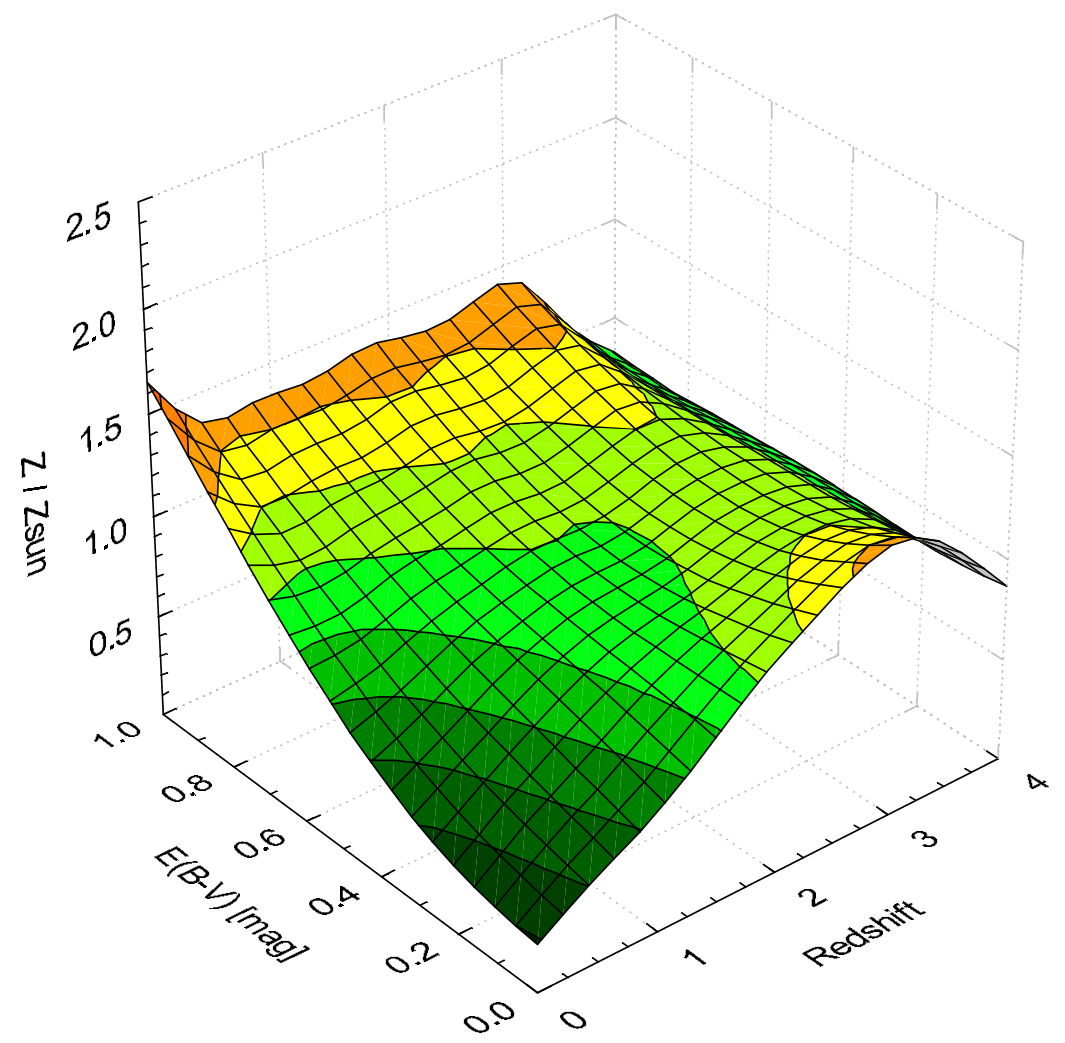


This figure "conti.fig10.gif" is available in "gif" format from: http://arxiv.org/ps/astro-ph/0307470v1 


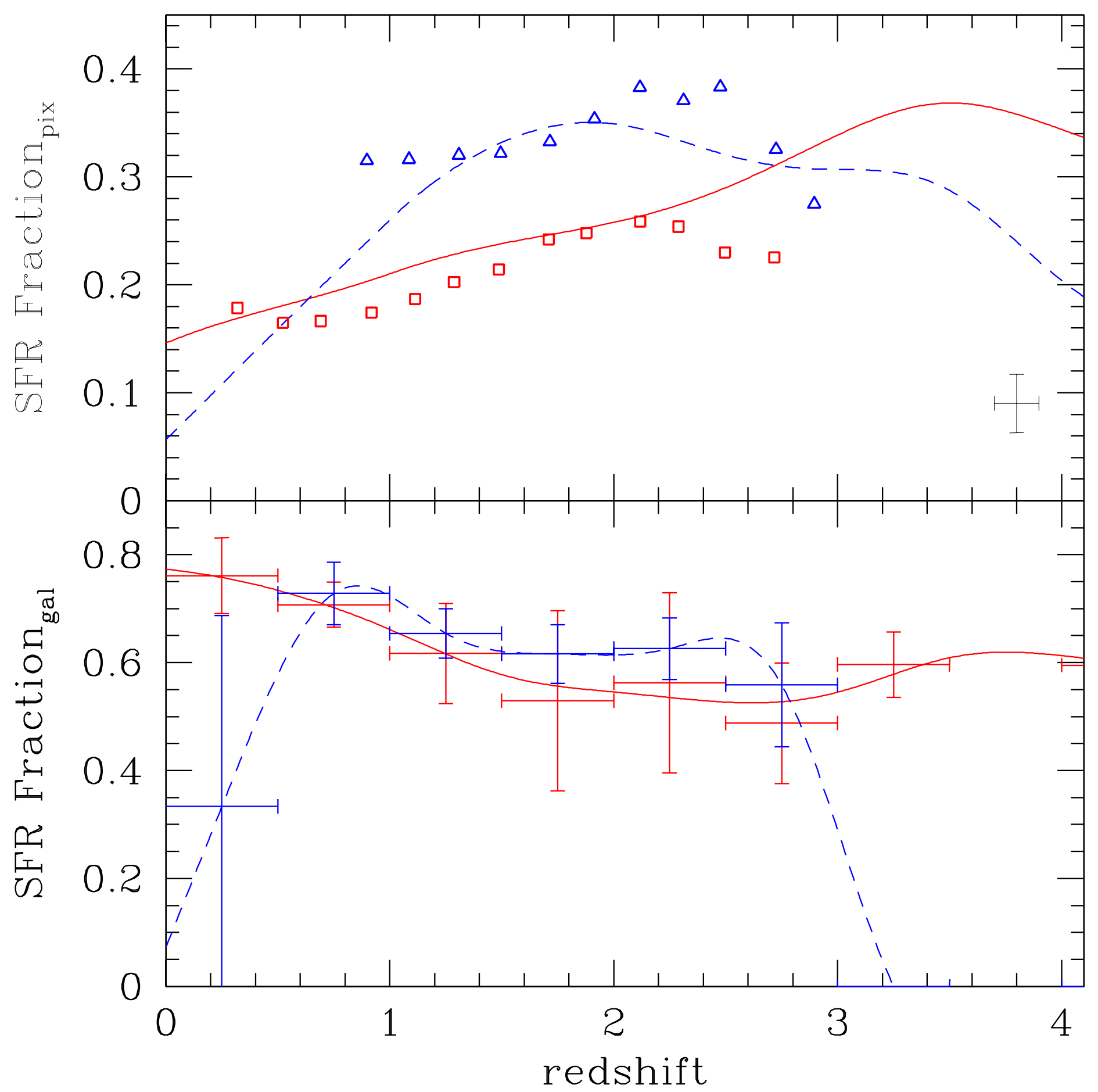




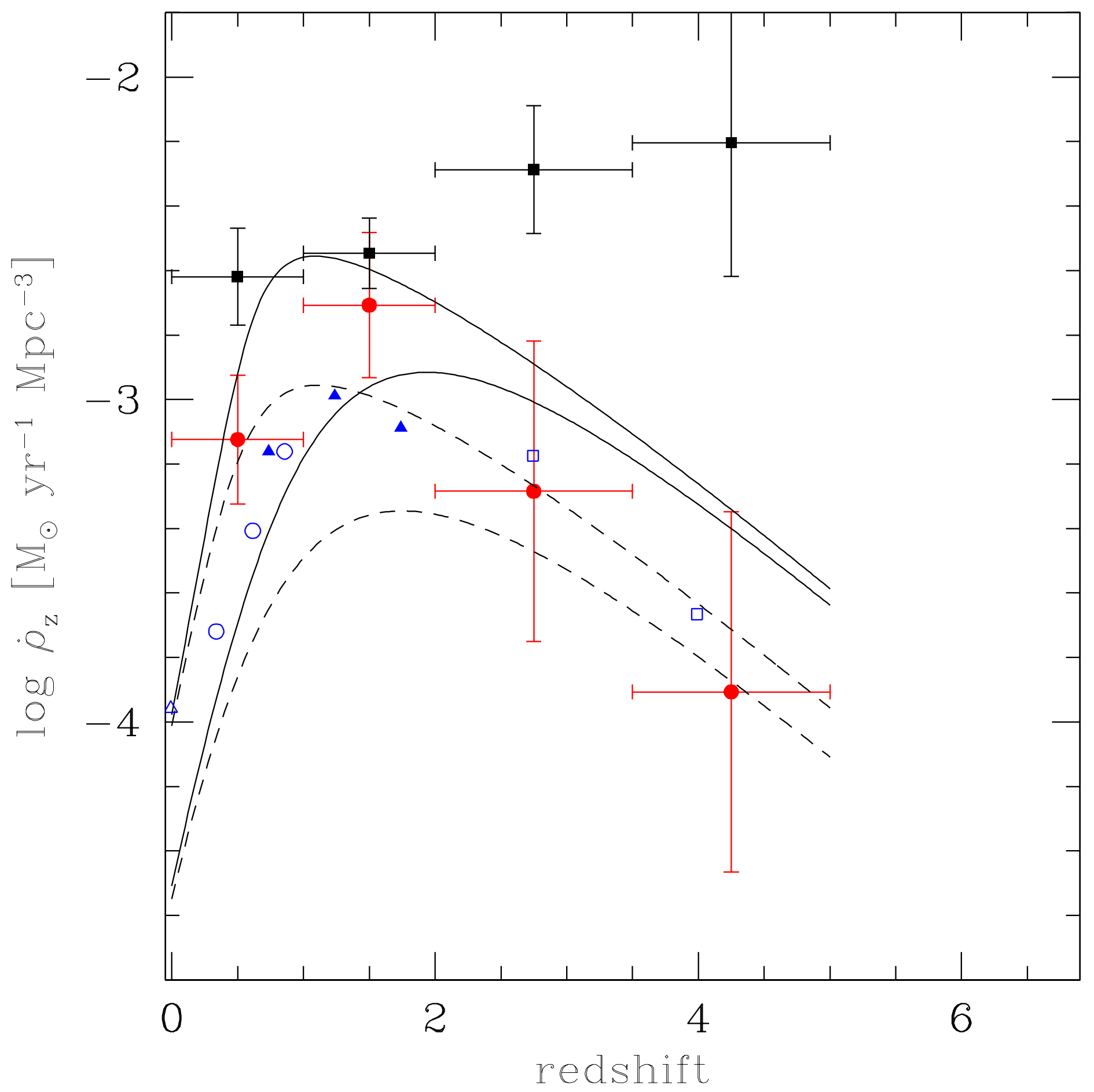

